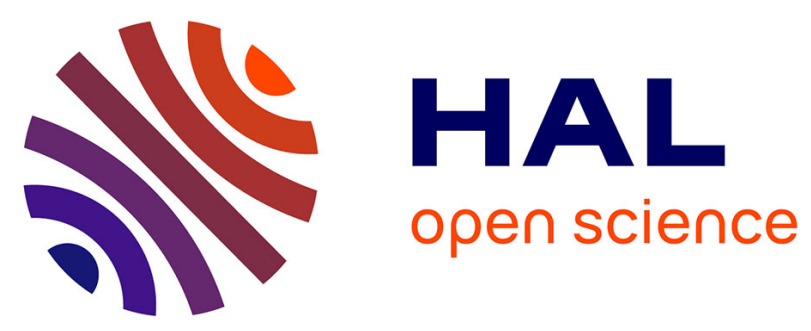

\title{
Synthesis and self-assembly of Xylan-based amphiphiles: from bio-based vesicles to antifungal properties
}

Julien Rosselgong, Maud Chemin, Cédric Cabral Almada, Gauvin Hemery, Jean-Michel Guigner, Guillaume Chollet, Gilles Labat, Denilson da Silva Perez, Frédérique Ham-Pichavant, Etienne Grau, et al.

\section{To cite this version:}

Julien Rosselgong, Maud Chemin, Cédric Cabral Almada, Gauvin Hemery, Jean-Michel Guigner, et al.. Synthesis and self-assembly of Xylan-based amphiphiles: from bio-based vesicles to antifungal properties. Biomacromolecules, 2019, 20 (1), pp.118-129. 10.1021/acs.biomac.8b01210 . hal01906661

\section{HAL Id: hal-01906661 \\ https://hal.science/hal-01906661}

Submitted on 6 Nov 2018

HAL is a multi-disciplinary open access archive for the deposit and dissemination of scientific research documents, whether they are published or not. The documents may come from teaching and research institutions in France or abroad, or from public or private research centers.
L'archive ouverte pluridisciplinaire HAL, est destinée au dépôt et à la diffusion de documents scientifiques de niveau recherche, publiés ou non, émanant des établissements d'enseignement et de recherche français ou étrangers, des laboratoires publics ou privés. 


\title{
Synthesis and self-assembly of Xylan-based amphiphiles:
}

\section{From bio-based vesicles to antifungal properties}

Julien Rosselgong ${ }^{1,2}$, Maud Chemin ${ }^{1,2}$, Cédric Cabral Almada ${ }^{1,2}$, Gauvin Hemery ${ }^{1,2}$, JeanMichel Guigner ${ }^{3}$, Guillaume Chollet ${ }^{4}$, Gilles Labat ${ }^{5}$, Denilson Da Silva Perez ${ }^{5}$, Frédérique Ham-Pichavant ${ }^{1,2}$, Etienne Grau ${ }^{1,2}$, Stéphane Grelier ${ }^{1,2^{*}}$, Sébastien Lecommandoux ${ }^{1,2^{*}}$, Henri Cramail $^{1,2 *}$

${ }^{1}$ CNRS, LCPO, UMR 5629, 16 av. Pey Berland, Pessac, F-33600, France

${ }^{2}$ Univ. Bordeaux, LCPO, UMR 5629, 16 av. Pey Berland, Pessac, F-33600, France

${ }^{3}$ Institut de Minéralogie, de Physique des Matériaux et de Cosmochimie (IMPMC), Sorbonne Université, UMR CNRS 7590, MNHN, IRD UR 206, 75252 Paris cedex 05, France

${ }^{4}$ ITERG, 11 rue Gaspard Monge, Parc Industriel, Pessac cedex, F-33600, France

${ }^{5}$ FCBA, Institut technologique FCBA, 10 rue Galilée, Champs-sur-Marne, F-77420, France

* Correspondence: Prof. S. Lecommandoux (Lecommandoux@enscbp.fr); Prof. H. Cramail (Cramail@enscbp.fr); Prof. Stéphane Grelier (sgrelier@enscbp.fr)

\begin{abstract}
This work aims at designing functional biomaterials through selective chemical modification of xylan from beechwood. Acidic hydrolysis of xylan leaded to well-defined oligomers with an average of six xylose units per chain and with an aldehyde group at the reductive end. Reductive amination was performed on this aldehyde end-group to introduce an azide reactive group. 'Click chemistry' was then applied to couple these hydrophilic xylans moieties with different hydrophobic fatty acid methyl esters that were previously functionalized with complementary alkyne functions. The resulting amphiphilic bio-based conjugates were then self-assembled using three different methods, namely direct solubilization, thin-film rehydration/extrusion and microfluidics. Well-defined micelles and vesicles were obtained
\end{abstract}


and their high loading capacity with propiconazole as an antifungal active molecule was shown. The resulting vesicles loaded with propiconazole in a microfluidic process, proved to significantly improve the antifungal activity of propiconazole, demonstrating the high potential of such xylan-based amphiphiles.

\section{1- Introduction}

Knowing that the fossil fuel available on earth will be spent in the foreseeable future that the barrel price is quite volatile and because of environmental issues, the development of new chemicals from renewable resources is nowadays a strategic research area. Among the available renewable biomass, polysaccharides such as starch, pectin, cellulose and hemicelluloses, gums or arabinogalactan confer diverse functions of food reserve, cementing, ion balancing, structure, defense or cell adhesion [1]. Within the last two decades, there has been a growing interest in lignocellulosic biomass, which is a sustainable resource to produce fuels, chemicals and materials [2-4]. Contrarily to cellulose that is the most industrialized polysaccharide, woody hemicelluloses are less valued. The most abundant hemicellulosic polymers are xylans, essentially linear heteropolysaccharides, accounting for $25-35 \%$ of the dry biomass of woody tissues. Extensive literature data report on the isolation, characterization, derivatization and applications of xylans [5-9]. However, the chemical modification and the degradation of hemicelluloses into oligosaccharides are still a challenging task that would open the route to biomacromolecular engineering.

Amphiphilic copolymers are composed of hydrophilic and hydrophobic segments that confer the behavior of self-assembly to these systems. Such materials can self-organize into nanoscale-size objects such as micelles, wormlike micelles or vesicles in solution, can form some well-defined assemblies at interfaces such as air-liquid, liquid-solid or air-solid and have been studied in the past recent years as nanosized reactors, nano-patterned surfaces for lithography, organic-inorganic mesostructures and drug delivery systems [10]. Polysaccharides-based amphiphiles are of particular interest due to their biocompatibility, bioactivity and biodegradability. Polysaccharides-based block copolymers can be designed using different methods, such as enzymatic polymerization, ring-opening polymerization, reversible-deactivation radical polymerization or end-to-end coupling [11]. In the last case, many oligosaccharides from amylose [12], cellulose [13], chitosan [14], dextran [15], maltoheptaose [16], xyloglucan [17], hyaluronan [18], galactan [19] and others have been 
coupled to synthetic blocks, making such bioconjugates of major interest especially for biomedical applications.

The aim of this contribution is to develop high added-value xylan derivatives, by designing fully bio-based amphiphilic oligomers. Beechwood xylans were first degraded into xylooligosaccharides by acidic hydrolysis [9]. The hydrolysis conditions were previously optimized in order to obtain well-defined xylo-oligosaccharides, i.e. oligomeric chains of about six xylose units that have only one methylglucuronic acid unit positioned at the nonreductive chain end [20-21]. The xylo-oligomers were then functionalized from their reductive end with an azide moiety, providing them the ability to couple with alkynefunctionalized fatty acid derivatives using 'click chemistry'. The obtained amphiphilic bioconjugates were finally studied according to their self-assembly properties that were demonstrated using three different methods: direct solubilization, thin-film rehydration followed by extrusion and microfluidics. Thereafter the resulting amphiphilic self-assembled nanostructures were characterized via dynamic light scattering (DLS), static light scattering (SLS) and transmission electron microscopy (TEM). Loading experiments were conducted by microfluidics to encapsulate either nile red, further detected by confocal microscopy or propiconazole to undergo antifungic biological evaluation. High loading capacity, together with significant improvement of the antifungal activity of propiconazole were demonstrated.

\section{2- Experimental}

\section{Materials}

Xylans from beechwood (MGX) were purchased from Symrise and ultrafiltrated prior reaction [20]. Methyl oleate (MeOl, 83.5\%) and methyl ricinoleate (MeRic, 90.9\%) were donated by ITERG and used without further purifications. Copper(II) sulfate and baryum hydroxide $\left(\mathrm{Ba}(\mathrm{OH})_{2}\right)$ were obtained from Prolabo. Cuprisorb was purchased from Seachem. Ethanol (96.0\%, EtOH) was obtained from VWR international. Propiconazole (solution 100 $\mathrm{ng} / \mu \mathrm{l}$ in $\mathrm{MeOH}$ ), sulfuric acid (ACS reagent, 95.0-98.0\%, $\mathrm{H}_{2} \mathrm{SO}_{4}$ ), 2-aminoethyl chloride (99.0\%), sodium cyanoborohydride (95\%), sodium azide (reagent plus, $\geq 99.5 \%$ ), propargyl alcohol (99\%), 1,5,7-triazabicyclo[4.4.0]dec-5-ene (98\%, TBD), nile red (for microscopy), ethyl acetate, magnesium sulfate, dimethyl sulfoxide (DMSO), sodium L-ascorbate and tetrahydrofuran (THF) were purchased from Sigma Aldrich and used without further purification. Dialysis was conducted using a Spectra/Por®6 MWCO 100-500 Da membrane. 


\section{Synthesis procedures}

Dilute acidic hydrolysis of xylan. A scale up experiment, times 120 for the comparison of mass of MGX involved, was performed compared to the acidic hydrolysis described previously [20-21] and is detailed in the supplementary information.

Reductive amination and functionalization with sodium azide. The overall reaction allowing the functionalization of the XOS with an azide group is shown in Scheme 1A. The first step includes a reductive amination of the aldehyde present at the terminal end of the XOS following an adapted procedure [22]. An amount of $10 \mathrm{~g}$ of XOS was dissolved in deionized water at $100 \mathrm{~g} . \mathrm{L}^{-1}$. 2-chlorethylamine hydrochloride (15 Eq.) was added under stirring. The mixture was stirred at $50^{\circ} \mathrm{C}$ for 3 days and $\mathrm{NaBH}_{3} \mathrm{CN}$ was added in 3 portions ( 3 * 5 Eq.) every day. After cooling down, the mixture was precipitated 3 times in EtOH, resulting in $6.84 \mathrm{~g}$ of brown powder after freeze-drying. In a second step, the obtained XOS$\mathrm{NH}-\left(\mathrm{CH}_{2}\right)_{2}-\mathrm{Cl}$ was dissolved in $40 \mathrm{~mL}$ of deionized water and reacted overnight with sodium azide (4 Eq.) at $80^{\circ} \mathrm{C}$. The mixture was first cooled down to room temperature then to $0^{\circ} \mathrm{C}$ with an ice bath. An amount of $3.7 \mathrm{~g}$ of $\mathrm{KOH}$ was added to the mixture which was then allowed to stir for another hour at $0^{\circ} \mathrm{C}$ [23]. The final product, XOS-NH- $\left(\mathrm{CH}_{2}\right)_{2}-\mathrm{N}_{3}$, was purified by precipitation in $\mathrm{EtOH}$, solubilized in deionized water then freeze-dried yielding $6.84 \mathrm{~g}$ of a pale brown powder.

Transesterification of fatty acid methyl ester. Both $\mathrm{MeOl}$ and MeRic fatty acid methyl esters (FAME) were functionalized with an alkyne group following the synthesis depicted in Scheme 2B. In a typical transesterification procedure, 10 Eq. of propargyl alcohol and $0.1 \mathrm{Eq}$. of TBD were added to $10 \mathrm{~g}$ of $\mathrm{MeOl}$. The mixture was stirred at $100^{\circ} \mathrm{C}$ for 4 hours under a gentle flux of $\mathrm{N}_{2}$. To follow, the mixture was placed for 4 hours at $100^{\circ} \mathrm{C}$ under dynamic vacuum. After cooling down to room temperature, $200 \mathrm{~mL}$ of ethyl acetate where added to the mixture followed by 4 consecutive washes with $50 \mathrm{~mL}$ of deionized water. The organic phase was dried using $\mathrm{MgSO}_{4}$, filtered, then finally dried under high vacuum to yield $7.5 \mathrm{~g}$ of pale yellow oil of alkyne functionalized $\mathrm{MeOl}$.

Huisgen cycloaddition. Amphiphilic bioconjugates were produced by 'click' reactions between azide functionalized XOS and alkyne functionalized FAME via the Huisgen cycloaddition as presented in Scheme 1C. In a typical Huisgen cycloaddition, $2 \mathrm{~g}$ of XOS azide functionalized were added in a $20 \mathrm{~mL}$ solution of DMSO and let under stirring 
overnight at $30^{\circ} \mathrm{C}$. Alkyne functionalized FAME, for example MeRic (0.627 g, 1 Eq.) was added to the mixture. After $15 \mathrm{~min}$, the sodium ascorbate $(0.369 \mathrm{~g}, 1 \mathrm{Eq}$.) was introduced in the reaction mixture followed by the addition of $\mathrm{CuSO}_{4}(0.292 \mathrm{~g}, 1 \mathrm{Eq}$. $) 15 \mathrm{~min}$ later. The reaction was allowed to stir for 24 hours at $30^{\circ} \mathrm{C}$. Yet, $20 \mathrm{~mL}$ of deionized water was added to the mixture with $1.168 \mathrm{~g}$ of Cuprisorb (4 weight eq. of $\mathrm{CuSO}_{4}$ ) [24], a powerful copper absorbent, and stirred overnight. Cuprisorb beads were separated by centrifugation ( $3800 \mathrm{rpm}$, $5^{\circ} \mathrm{C}, 25 \mathrm{~min}$ ) and the reaction solution was dialyzed (MWCO: 100-500 Da) for 4 days against deionized water then freeze-dried to afford $0.689 \mathrm{~g}$ of pale green powder (yield $\approx 25 \%$ ).

\section{Self-assembly procedures}

Direct solubilization. Bioconjugates obtained after 'click' reaction between azide functionalized XOS and alkyne functionalized FAME, either XOS- $b$-MeOl or XOS- $b$-MeRic depending on the FAME used, were dispersed in filtered deionized water (using a $0.45 \mu \mathrm{m}$ cellulose acetate filter) or in filtered chloroform (using a $0.45 \mu \mathrm{m}$ PTFE filter) at a concentration of $10 \mathrm{mg} \cdot \mathrm{mL}^{-1}$, then diluted to $1 \mathrm{mg} \cdot \mathrm{mL}^{-1}$ and $0.1 \mathrm{mg} \cdot \mathrm{mL}^{-1}$ using filtered solvents. Solutions were magnetically stirred for 24 hours at room temperature and filtered through cellulose acetate or PTFE filters without significant loss prior size characterization by DLS, SLS and TEM.

Thin-film hydration method followed by extrusion. An amount of $50 \mathrm{mg}$ of bioconjugates XOS- $b$-MeOl and XOS- $b$-MeRic was dispersed in filtered THF at a concentration of $10 \mathrm{mg} \cdot \mathrm{mL}^{-1}$ and stirred overnight in a $50 \mathrm{~mL}$ round bottom flask. THF was removed from the flask via rotary evaporation followed by high vacuum to form a thin film. This film was rehydrated dropwise using $5 \mathrm{~mL}$ of filtered deionized water. The solution was then gently stirred at room temperature for 3 hours, then extruded back and forth 15 times through $0.4 \mu \mathrm{m}$ polycarbonate membranes prior size analysis by DLS.

Microfluidics method using Dolomite's micromixer system. Bioconjugates XOS- $b$ $\mathrm{MeOl}$ or XOS- $b$-MeRic were dispersed in THF at a concentration of $2 \mathrm{mg} \cdot \mathrm{mL}^{-1}$ and stirred overnight before filtration using a $0.45 \mu \mathrm{m}$ PTFE filter. The resulting solution was mixed with filtered deionized water using the Dolomite's micromixer system in order to create the selfassembled objects. This system was composed of a microfluidic device (Mitos micromixer chip, lamination-based compact glass microfluidic device), two precision pumps (Mitos-PPump flow control, one filled with the bioconjugate in THF solution and the other one with 
filtered deionized water), fluidic elements (linear connector 4-way and a $\mathrm{H}$ interface to enable fluidic connection between the tubing and chip), a microscope (Meros TCU-100) and software to achieve microfluidic mixing (flow control center) and to observe the mixing chip (capture OEM). The key component in the system was the micromixer chip that allowed rapid mixing of the two streams from low to high flow rate ratios $\left(50 \mu \mathrm{L} \cdot \mathrm{min}^{-1}<\right.$ flow rate $<1000$ $\left.\mu \mathrm{L} \cdot \mathrm{min}^{-1}\right)$. THF containing bioconjugates was mixed with filtered deionized water at different flow rates (from to 200 to $1000 \mu \mathrm{L} \cdot \mathrm{min}^{-1}$ ) and the resulting solutions were collected for $5 \mathrm{~min}$ then analyzed through light scattering methods. When changing one flow rate parameter, a waiting time of $1 \mathrm{~min}$ was respected before the resultant solution was started to be collected.

\section{Loading procedure}

Microfluidics method using Dolomite's micromixer system. For nile red loading, $300 \mu \mathrm{L}$ of a solution of nile red in acetone $\left(10^{-4} \mathrm{M}\right)$ were added to $3 \mathrm{~mL}$ of THF containing 6 $\mathrm{mg}$ of bioconjugate XOS- $b$-MeOl. The process of self-assembly used was similar as described above in using a flow rate of $200 \mu \mathrm{L} \cdot \mathrm{min}^{-1}$ of THF/acetone containing bioconjugate and nile red mixed with a flow rate of $600 \mu \mathrm{L} \cdot \mathrm{min}^{-1}$ of filtered deionized water. The sample obtained was purified by dialysis (MWCO: $1000 \mathrm{Da}$ ) for 2 days against deionized water prior light scattering and confocal microscopy analyses. For propiconazole loading, from $300 \mu \mathrm{L}$ to 1500 $\mu \mathrm{L}$ of a solution of propiconazole in methanol $(100 \mathrm{ng} / \mu \mathrm{L})$ were added to $4 \mathrm{~mL}$ of $\mathrm{THF}$ containing $8 \mathrm{mg}$ of bioconjugate XOS- $b$-MeOl. The process of self-assembly used was similar as described above in using a flow rate of $200 \mu \mathrm{L} \cdot \mathrm{min}^{-1}$ of $\mathrm{THF} / \mathrm{MeOH}$ containing bioconjugate and propiconazole mixed with a flow rate of $600 \mu \mathrm{L} \cdot \mathrm{min}^{-1}$ of filtered deionized water. The sample obtained was purified by dialysis (MWCO: $1000 \mathrm{Da}$ ) for 2 days against deionized water prior light scattering and antifungal biological evaluation.

\section{Characterization methods}

Nuclear Magnetic Resonance (NMR). Analyses were performed at $298 \mathrm{~K}$ on a Bruker AVANCE I apparatus equipped with a $5 \mathrm{~mm}$ probe operating at $400.2 \mathrm{MHz}$ for ${ }^{1} \mathrm{H}$. An amount of $20 \mathrm{mg}$ of material was dissolved/dispersed in $0.5 \mathrm{~mL}$ of deuterated solvent $\left(\mathrm{CDCl}_{3}\right.$, Eurisotop; 99.5 atom\%D, $\mathrm{D}_{2} \mathrm{O}$, Eurisotop; 99.9 atom\%D or DMSO-d6, Eurisotop; 99.8 atom\%D) and 128 scans were recorded for ${ }^{1} \mathrm{H}$ experiments, 4096 for ${ }^{13} \mathrm{C}$ experiments. The spectra obtained by proton-detected Heteronuclear Single-Quantum Correlation (HCQC) were acquired over a spectral width of $20,000 \mathrm{~Hz}$ for ${ }^{13} \mathrm{C}$ and $4,000 \mathrm{~Hz}$ for ${ }^{1} \mathrm{H}$ with 1024 data 
points; 256 increments collected and a delay between two pulses of $1.5 \mathrm{~s}$. Spectra were analyzed using Bruker Topspin software.

Size Exclusion Chromatography (SEC). SEC analyses of MGX and XOS were performed on a Spectra-Physics Analytical system equipped with a TSK PWH $13 \mu \mathrm{m}$ guard column, a TSK gel G2000PW column and a TSK gel G3000PW column along with a refractive index detector (model RID-10A) from Shimadzu. The system was calibrated with 6 Shodex pullulan standards ranging from $5 \cdot 9 \cdot 10^{3}$ to $375.10^{3}$ g.mol ${ }^{-1}$ and one oligosaccharide laminarihexaose from Megazyme with $\mathrm{M}=990.9$ g.mol ${ }^{-1}$. A phosphate eluent was used $\left(\mathrm{NaNO}_{3} 0.2 \mathrm{M}, \mathrm{Na}_{2} \mathrm{HPO}_{4} 0.01 \mathrm{M}\right.$ adjusted to $\left.\mathrm{pH}=9\right)$ and running at $0.5 \mathrm{~mL} \cdot \mathrm{min}^{-1}$. SEC analyses of FAME and their alkyne functionalized entities were performed on an Ultimate 3000 system from Thermoscientific, equipped with three G2000, G3000 and G4000 TOSOH HXL gel columns $(300 \times 7.8 \mathrm{~mm})$ (exclusion limits from $1000 \mathrm{Da}$ to $400000 \mathrm{Da}$ ) at a flowrate of $1 \mathrm{~mL} \cdot \mathrm{min}^{-1}$. Columns temperature was held at $40^{\circ} \mathrm{C}$. The system also includes a multi-angle light scattering detector, MALLS and differential refractive index detector, dRI, from Wyatt technology. THF was used as the eluent and polystyrenes as standards.

Matrix Assisted Laser Desorption/Ionisation-Time of flight (MALDI-TOF). MALDI-TOF analyses were performed on a Voyager apparatus (Applied Biosystems) equipped with a laser pulsed $\mathrm{N}_{2}(337 \mathrm{~nm})$ and an ionisation source assisted by a matrix with a time of flight analyser. Spectra were recorded in positive mode. Samples or 2,5dihydroxybenzoic acid matrix (FlukaChemika; 2,5-DHB > 98.5\%) were solubilised in deionised water at $10 \mathrm{mg} \cdot \mathrm{mL}^{-1}$. Then, solutions were prepared at a volume ratio matrix: sample 10:1. Finally, 1 or $2 \mu \mathrm{L}$ of the prepared solutions were deposited on a plate that was dried under vacuum prior analysis.

Dynamic Light Scattering (DLS) and Static Light Scattering (SLS). Dynamic Light Scattering (Malvern DLS) was used to obtain particle average sizes and their size distribution by using a Malvern ZetaSizer Nano ZS instrument equipped with a standard $\mathrm{HeNe}$ laser emitting at $632.8 \mathrm{~nm}$. As a first approach, measurements were carried out with this device at a single angle of $90^{\circ}$ and at $25^{\circ} \mathrm{C}$. Particle sizes and their size distribution were determinate by the cumulative method (second order). Appropriated dispersants were selected $\left(\mathrm{H}_{2} \mathrm{O}, \mathrm{CHCl}_{3}\right.$ or various $\mathrm{THF} / \mathrm{H}_{2} \mathrm{O}$ mixtures $)$ depending on the self-assembly procedure. Moreover, to accurately determine the gyration radius $(\mathrm{Rg})$ and the hydrodynamic radius $\left(\mathrm{R}_{\mathrm{H}}\right)$ 
of the nano-objects formed by the bioconjugates, multi-angle light-scattering analysis (ALV DLS) was performed using an ALV laser goniometer, with a $22 \mathrm{~mW}$ linearly-polarised laser $(632.8 \mathrm{~nm} \mathrm{HeNe})$ and an ALV-5000/EPP multiple tau digital correlator (125 ns initial sampling time). All the measurements were performed at a constant temperature of $25^{\circ} \mathrm{C}$. The accessible scattering angles range from 30 to $150^{\circ}$. The solutions were placed in $20 \mathrm{~mm}$ diameter glass cells. Data were acquired using the ALV correlator control software, and the counting time was fixed for each sample at $15 \mathrm{~s}$ for dynamic diffusion measurements. The hydrodynamic radius was calculated from the diffusion coefficient using the Stokes-Einstein relation and the gyration radius was calculated from a Guinier plot.

Transmission Electron Microscopy (TEM). TEM images were acquired on a Hitachi H7650 microscope working at 80kV and equipped with an ORIUS SC1000 11MPx camera. Samples were prepared by direct deposition of a $1 \mathrm{mg} \cdot \mathrm{mL}^{-1}$ droplet on carbon grids (300 mesh $\mathrm{Cu}-300 \mathrm{LD}$ from Pacific Grid Tech.). For $\mathrm{CHCl}_{3}$, the droplet was allowed to dry entirely, while for $\mathrm{H}_{2} \mathrm{O}$, the liquid was removed after 2 min using a filter paper. After drying, a droplet of uranyl acetate $0.5 \%$ in water was deposited. After 2 min the rest of liquid was removed using a filter paper.

Cryo-Transmission Electron Microscopy (cryo-TEM). Cryo-TEM micrographs were obtained as following. A drop of suspension at a concentration of $6 \mathrm{mg} / \mathrm{mL}$ was deposited on a "quantifoil” $®($ Quantifoil Micro Tools GmbH, Germany) carbon membrane. The excess of liquid on the membrane was absorbed with a filter paper and the membrane was quench-frozen quickly in liquid ethane to form a thin vitreous ice film. Once placed in a Gatan 626 cryo-holder cooled with liquid nitrogen, the samples were transferred in the microscope and observed at low temperature $\left(-180^{\circ} \mathrm{C}\right)$. Cryo-TEM images were recorded on ultrascan 2k CCD camera (Gatan, USA), using a LaB6 JEOL 2100 (JEOL, Japan) cryo microscope operating at $200 \mathrm{kV}$ with a JEOL low dose system (Minimum Dose System, MDS) to protect the thin ice film from any irradiation before imaging and reduce the irradiation during the image capture.

Laser Scanning Confocal Microscopy (LSCM). LSCM images were acquired on an inverted Leica TCS SP5 microscope equipped with an HCX PL APO 63X, NA 1.4 oil immersion objective in fluorescence mode. The laser outputs were controlled via the Acousto- 
Optical Tunable Filter (AOTF) and one collection window using the Acousto-Optical Beam Splitter (AOBS) and photomultiplicators (PMT) as follows: Nile Red was excited with a laser at $561 \mathrm{~nm}(12.5 \%)$ and measured with emission settings at $571 \mathrm{~nm}-624 \mathrm{~nm}$. The Helium-Neon laser at $633 \mathrm{~nm}(14 \%)$ was only used in transmission mode. Images were collected in simultaneous mode using a resonant scanner at $8000 \mathrm{~Hz}$ in bidirectional mode at a resolution of $512 * 512$ pixels.

Antifungal biological evaluation. Fungal strains used in this study are part of the FCBA collection from Bordeaux. $10 \mathrm{~mL}$ of bioconjugate XOS- $b$-MeOl loaded with propiconazole solutions at several concentrations were filtered with $0.2 \mu \mathrm{m}$ PES sterile filters then added to $65 \mathrm{~mL}$ of malt/agar $(4 \% / 2 \%)$. The final solutions were then split in three plates and experiments were performed in three independent biological repeats were the strain Trametes versicolor (CTB $863 \mathrm{~A}$ ) was propagated and incubated at $22^{\circ} \mathrm{C}$ and $70 \%$ of relative humidity. Radial growth assay was performed on malt/agar medium supplemented prior solidification with bioconjugate XOS- $b-\mathrm{MeOl}$ loaded with propiconazole. One plug of fungal mycelia was put on supplemented medium to evaluate the ability of bioconjugate XOS- $b$ $\mathrm{MeOl}$ loaded with propiconazole to affect fungal growth. Agar plates were incubated at $22^{\circ} \mathrm{C}$ and $70 \%$ of relative humidity until complete growth.

\section{3- Results and discussion}

\section{1- Synthesis procedures}

Dilute acidic hydrolysis of xylan. The production of well-defined XOS by mild sulfuric acid hydrolysis of beechwood xylans was recently reported by Chemin et al. [20-21] and its scale up is reproducible and allow the production of $75 \mathrm{~g}$ of XOS per batch.

Reductive amination and functionalization with sodium azide. The XOS formed by controlled hydrolysis of MGX were functionalized by reductive amination of the aldehyde chain-end followed by a nucleophilic substitution with sodium azide. Hence, an azide function was introduced to the extremity of the XOS (Scheme 2A). Figure SS1 shows the Fourier transform infrared (FTIR) spectra of the XOS before (black, bottom) and after (red, top) reductive amination followed by reaction with sodium azide: clearly, a specific band appears at $2100 \mathrm{~cm}^{-1}$ corresponding to the azide function [25]. Figure 1 represents the ${ }^{1} \mathrm{H}$ 
NMR spectra in $\mathrm{D}_{2} \mathrm{O}$ of XOS before (bottom) and after (top) reductive amination with 2aminoethyl chloride followed by reaction with sodium azide. The top spectrum reveals the appearance of new signals for the modified $\mathrm{XOS}$ at 2.4-2.8 ppm $\left(4 \mathrm{H},-\mathrm{CH}_{2}-\mathrm{CH}_{2}-\mathrm{N}_{3}\right)$ corresponding to the ethylazide chain-end. According to previous DOSY experiments, one can conclude that the azide moiety is really linked to the XOS chain-end [20].

Transesterification of fatty acid methyl ester. Vegetable oils are easily accessible and in large quantities. Many articles and reviews deal with the functionalization of triglycerides and fatty acids for the synthesis of bio-sourced polymers [26-33]. The transesterification of fatty esters is a catalyzed reaction generally carried out in the bulk at rather high temperature $\left(120^{\circ} \mathrm{C}\right)$ [34-35]. In this study, the methyl oleate $(\mathrm{MeOl})$ and the methyl ricinoleate (MeRic) were used as mono-unsaturated $\mathrm{C} 18$ fatty esters as the latter can be produced with a relatively high purity and only differ by the presence or not of one $\mathrm{OH}$ group linked to the fatty acid backbone. These substrates were functionalized by transesterification reaction with propargyl alcohol in the presence of TBD used as a catalyst (Scheme 1B) [36] to yield fatty esters bringing an alkyne end-group that could be further engaged in coupling reactions with XOS-NH- $\left(\mathrm{CH}_{2}\right)_{2}-\mathrm{N}_{3}$, giving access to bio-sourced and amphiphilic bioconjugates. Due to the relatively low boiling point of propargyl alcohol $\left(115^{\circ} \mathrm{C}\right)$, a large excess of the latter was used (10 equivalents) and the reaction temperature was decreased to $100^{\circ} \mathrm{C}$. These two experimental parameters have the advantage of limiting the auto condensation of MeRic by transesterification on itself. ${ }^{1} \mathrm{H}$ NMR analyses, presented in Figure 2A (MeOl) and Figure 2C (MeRic), clearly show that the conversion is complete with the disappearance of the $-\mathrm{CH}_{3}$ of the ester at $3.62 \mathrm{ppm}$ and the appearance of the protons resulting from the propargyl alcohol at $2.4 \mathrm{ppm}(\mathrm{H} \equiv)$ and $4.6 \mathrm{ppm}\left(-\mathrm{CH}_{2}-\mathrm{O}\right)$. SEC analyses in THF, presented in Figure 3B (MeOl) and Figure 3D (MeRic), show a slight increase in molar mass (from 480 g.mol ${ }^{-1}$ to 500 g.mol ${ }^{-1}$ for $\mathrm{MeOl}$ and from 450 g.mol ${ }^{-1}$ to 480 g.mol ${ }^{-1}$ for MeRic) and no signal attributable to a possible polymerization side-reaction.

Huisgen cyclo-addition. 'Click chemistry' was reported as a concept of 'green chemistry' in 2001 by Kolb et al. [37]. The reactions are characterized by presenting a good selectivity, a simple experimental device with a minimum of synthetic steps, aqueous and oxygen application, tolerance to numerous chemical groups and quantitative yields. A widely used 'click' reaction is typically the azide-alkyne cyclo-addition catalyzed with $\mathrm{Cu}$ (I) or 
Huisgen cyclo-addition. The concept has further been extended to other reactions with, for example, the thiol-ene addition, the Diels-Alder cyclo-addition or the thiol-yne addition [38]. Herein, the XOS-NH- $\left(\mathrm{CH}_{2}\right)_{2}-\mathrm{N}_{3}$ were coupled with both fatty ester derivatives alkynefunctionalized through an azide-alkyne cyclo-addition, as presented in Scheme 1C. This reaction was carried out accordingly to a previously reported protocol [39]. The reaction solvent DMSO was chosen as it solubilizes well both hydrophilic and hydrophobic segments but also because it is the only solvent to fully solubilize the final amphiphilic bioconjugate soformed. The amount of catalytic system $\mathrm{CuSO}_{4} /$ sodium ascorbate was reduced to 1 equivalent compared to azide or alkyne functions, as its removal is the limiting factor of the purification step and an excess of it was not necessary to achieve a full conversion. FTIR spectra presented in Figure S6 shows the XOS azide functionalized and the bioconjugate XOS- $b$ $\mathrm{MeOl}$ after the 'click' reaction: the total disappearance of the characteristic azide band at $2100 \mathrm{~cm}^{-1}$ in the bioconjugate proves that the 'click' reaction was total. Cuprisorb was used in the purification process before the dialysis step because it is known to be a powerful copper absorbant [24]. The final purification step of dialysis is probably the limiting factor for the overall reaction as the relatively small bioconjugate obtained, around 1,300 g.mol ${ }^{-1}$, is susceptible to go through the dialysis membrane. The final bioconjugates were analyzed by ${ }^{1} \mathrm{H}$ NMR in DMSO-d6 (Figure 4) after freeze-drying. Both spectra, for XOS- $b-\mathrm{MeOl}$ and XOS- $b$-MeRic, show the characteristic peaks of the FAME, at 0.5-2.5 ppm, of the XOS at 3$5.5 \mathrm{ppm}$ and the triazole peak at $8.1 \mathrm{ppm}$ confirming the expected structure of the amphiphilic bioconjugates.

${ }^{1} \mathrm{H}$ NMR analyses in $\mathrm{D}_{2} \mathrm{O}$, selective solvent of the XOS segment and in $\mathrm{CDCl}_{3}$, selective solvent of the fatty acid segment, gave a first insight on the amphiphilic nature of the bioconjugates as shown in the Figure S7 and S8. It is revealed that in $\mathrm{D}_{2} \mathrm{O}$ mainly the domain of chemical shift belonging of the XOS is resolved and in $\mathrm{CDCl}_{3}$, only the domain of chemical shift corresponding to the fatty acid is resolved. This indicates that the two solvents are selective of a self-assembly structure that hides the hydrophobic groups of the FAME in $\mathrm{D}_{2} \mathrm{O}$ and the hydrophilic group of the XOS in $\mathrm{CDCl}_{3}$. This self-assembly process is discussed in the following part of the manuscript.

\section{2- Self-assembly properties}

As described in the experimental part, the self-assembly behavior of the two amphiphilic bioconjugates XOS- $b$-MeOl and XOS- $b$-MeRic was studied using three different methods such as direct solubilization, thin-film rehydration and microfluidics, each method 
having its own advantages and drawbacks. The resulting self-assembled objects were characterized in size and shape using four techniques: a routine single-angle dynamic light scattering at $90^{\circ}$ (Malvern DLS), a multi-angle light scattering (ALV DLS and static light scattering SLS), a transmission electron micrograph (TEM) or a cryo-transmission electron microscope (cryo-TEM) allowing to determine, respectively, particle sizes and their distribution (Malvern DLS), hydrodynamic $\left(\mathrm{R}_{\mathrm{H}}\right)$ and gyration radii $(\mathrm{Rg})$ and the nano-object shape defined by the ratio of the two previous radius (ALV DLS), the shape and size of the dry nano-objects (TEM) and finally the shape and size of the nano-objects directly in aqueous conditions (cryo-TEM). Subsequently, laser scanning confocal microscopy (LSCM) was used to determine the incorporation of the nile red dye in the nano-objects.

Direct solubilization. Both bioconjugates XOS- $b-\mathrm{MeO}$ and XOS- $b$-MeRic were solubilized either in water or in chloroform at a concentration of $10 \mathrm{mg} \cdot \mathrm{mL}^{-1}$ and then diluted to $1 \mathrm{mg} \cdot \mathrm{mL}^{-1}$ then $0.1 \mathrm{mg} \cdot \mathrm{mL}^{-1}$. Particle sizes obtained through DLS measurements are compiled in Table 1 and in Table S1.

For the bioconjugates dissolved in water, their hydrodynamic radii $\left(\mathrm{R}_{\mathrm{H}}\right)$ measured at $90^{\circ}$ range from $87 \mathrm{~nm}$ to $106 \mathrm{~nm}(0.163 \leq \mathrm{PDI} \leq 0.193)$ for the XOS- $b-\mathrm{MeOl}$ bioconjugate and from $88 \mathrm{~nm}$ to $91 \mathrm{~nm}(0.192 \leq \mathrm{PDI} \leq 0.230)$ for the XOS- $b$-MeRic bioconjugate with, in every cases, a monomodal size distribution. For both bioconjugates, the $\mathrm{R}_{\mathrm{H}}$ values are relatively stable within the range of concentrations studied with some relatively high PDI. Particles are still detected at the lowest concentration, meaning that $0.1 \mathrm{mg} \cdot \mathrm{mL}^{-1}$ is above the critical aggregation concentration. Furthermore, from SLS and multi-angle light scattering measurements (ALV) performed at a concentration of $1 \mathrm{mg} \cdot \mathrm{mL}^{-1}$, a $\mathrm{R}_{\mathrm{g}}=91 \mathrm{~nm}$ and a $\mathrm{R}_{\mathrm{H}}=$ $104 \mathrm{~nm}$ were obtained for the XOS- $b-\mathrm{MeOl}$ system and $\mathrm{a} \mathrm{R}_{\mathrm{g}}=66 \mathrm{~nm}$ and $\mathrm{R}_{\mathrm{H}}=61 \mathrm{~nm}$ for the XOS- $b$-MeRic system. In both cases, the ratio $\mathrm{R}_{\mathrm{g}} / \mathrm{R}_{\mathrm{H}} \approx 1$ suggests a vesicular morphology of the self-assembled nano-object [40-42]. This was confirmed by TEM analyses, as presented in Figure 6, where spherical objects with a hole in the center can be observed: at high magnification, the membrane of the vesicles can be detected. Higher resolution imaging was achieved when using cryo-TEM: the nano-objects formed could be observed in aqueous solution as shown in Figure 7 (A-D) and vesicular shape was confirmed with an accurate definition of the membrane of the vesicle. Nevertheless, the TEM and cryo-TEM pictures show similarly a high size polydispersity of the nano-objects, confirming the obtained results through DLS measurements at $90^{\circ}$ (Malvern) with a $\mathrm{PDI} \approx 0.2$. 
For the chloroform system, only the highest concentration of $10 \mathrm{mg} \cdot \mathrm{mL}^{-1}$ was analyzed through DLS measurements at $90^{\circ}$ (Malvern). When solubilizing XOS- $b$-MeOl and XOS- $b$ MeRic in this solvent, aggregates are formed and removed after filtration so that the two lowest concentrations of $1 \mathrm{mg} \cdot \mathrm{mL}^{-1}$ and $0.1 \mathrm{mg} \cdot \mathrm{mL}^{-1}$ could not be distinguished by lack of signal. The nano-objects detected at $10 \mathrm{mg} \cdot \mathrm{mL}^{-1}$ have a $\mathrm{R}_{\mathrm{H}}=74 \mathrm{~nm}$ for the XOS- $b-\mathrm{MeOl}$ and a $\mathrm{R}_{\mathrm{H}}=89 \mathrm{~nm}$ for the XOS- $b$-Ric with, in both cases, relatively high PDI $\approx 0.18$. The selfassembly properties in chloroform were not further exploited.

Thin-film hydration method followed by extrusion. This method was originally developed for liposomes preparation. It involves first the formation a thin lipid film in a round-bottom flask by rotary evaporation of an organic solvent. Upon the slow addition, then agitation of the dispersion medium (typically water of a buffer), heterogeneous liposomes (different size and multi-lamellar) are formed. Then, after extrusion through polycarbonate membranes, homogeneous small liposomes are obtained [43]. Even if not a perfect solvent for the systems XOS- $b$-MeOl and XOS- $b$-MeRic, we chose THF to disperse the bioconjugates and create the thin-film because of its low boiling point $\left(66^{\circ} \mathrm{C}\right)$. A full solubilization of the bioconjugates could have been obtained only in DMSO however this solvent would not be removed to create the thin film due to its high boiling point $\left(189^{\circ} \mathrm{C}\right)$. Upon addition of water, large nano-objects were formed, up to $128 \mathrm{~nm}$ for the XOS- $b$-MeOl system and $189 \mathrm{~nm}$ for the XOS- $b$-MeRic system, as evidenced by DLS measurements (see Table 2, entries 1 \& 3). However, their distribution is multimodal showing three peaks with accordingly very high $\mathrm{PDI}=0.35$ for the XOS- $b-\mathrm{MeOl}$ system and PDI $=0.38$ for the XOS- $b$-MeRic system. Extrusion through polycarbonate membrane followed the rehydration process and was performed through a $0.4 \mu \mathrm{m}$ membrane. The solutions containing a multimodal distribution of nano-objects were forced to pass through the membranes back and forth 15 times by applying a relatively high pressure. The obtained solutions after extrusion presented nanoparticles with lower particle sizes, i.e. $74 \mathrm{~nm}$ for the XOS- $b$-MeOl system and $86 \mathrm{~nm}$ for the XOS- $b$-MeRic system (see Table 2, entries $2 \& 4$ ). Moreover, their distributions became monomodal, even if the PDI stayed relatively high: PDI $=0.23$ for the $\mathrm{XOS}-b-\mathrm{MeOl}$ system and $\mathrm{PDI}=0.28$ for the XOS- $b$-MeRic system.

For an analysis by multi-angle light scattering, the initial solutions after rehydration were too polydisperse to be investigated properly. However, the $\mathrm{R}_{\mathrm{g}}$ and $\mathrm{R}_{\mathrm{H}}$ could be measured for the extruded solutions, giving a set of data presented in Table 2, entry 2 for the XOS- $b$-MeOl and entry 4 for the XOS- $b$-MeRic. The obtained results for these 2 solutions were very similar, 
giving a shape factor $\mathrm{R}_{\mathrm{g}} / \mathrm{R}_{\mathrm{H}} \approx 1$ in all cases, meaning that the shape of the nano-objects formed was vesicular with a radius being around $45 \mathrm{~nm}$. The sample $\mathrm{XOS}-b-\mathrm{MeOl}$ was also analyzed by cryo-TEM as shown in Figure 7 (E, F) and once again vesicular shape could clearly be observed with sizes and PDI confirming the light scattering experiments.

Microfluidic micromixer experiments. Due to optimized and stable mixing parameters, the preparation of self-assembled nano-objects via microfluidic systems may provide nanostructures with a better control over size in a high throughput manner. Such method used for the preparation of polymersomes (vesicles) was recently reviewed [44]. The first approaches described generating polymersomes using double emulsion microfluidics produced giant vesicles in the micrometer range [45-46]. Using very fast and controlled mixing processes, Prud'homme first evidenced the ability to form small size and reproducible polymer vesicles [47-48]. Later, the use of micromixer devices, as presented by Maskos and coll., enabled control over size and shape of polymersomes below 100 nanometers [49-50]. Recently, Le Fer et al. [51] reported the self-assembly of amphiphilic copolypeptide containing an Elastin-like polypeptide copolymerized with an $\gamma$-benzyl- $L$-glutamate block using the Dolomite micromixer chip, similar system used in the present study. This system allowed the fast mixing of at least two sources of different solutions in a laminar flow, permitting to vary the flow rate (D) of each solution and obtaining in the final mixed solution different solvent ratios (SR). We chose to mix a solution of THF containing the bioconjugate at a concentration of $2 \mathrm{mg} \cdot \mathrm{mL}^{-1}$ with water to obtain the self-assembled nano-objects. In the first set of experiments, we increased the flow rate of THF containing the bioconjugate $\left(\mathrm{D}_{\mathrm{THF}}\right)$ with respect to the flow rate of water $\left(\mathrm{D}_{\mathrm{H} 2 \mathrm{O}}\right)$ with a factor of 2 between $\mathrm{D}_{\mathrm{THF}}$ and $\mathrm{D}_{\mathrm{H} 2 \mathrm{O}}$ in order to keep a final SR equal to 2 . The final solutions were analyzed by DLS at $90^{\circ}$ in order to evaluate the $\mathrm{R}_{\mathrm{H}}$ of the nano-objects. The results are compiled in Table S2 (entries 11-16). In comparison with the two methods described previously, the PDI of the nano-objects formed was very low $(0.04<$ PDI $<0.08)$. However, no special trend could be observed for the size of the nano-objects formed (Table S2). In a second set of experiments, the flow rate $\mathrm{D}_{\mathrm{THF}}$ was kept constant at $200 \mu \mathrm{L} \cdot \mathrm{min}^{-1}$ while the flow rate $\mathrm{D}_{\mathrm{H} 2 \mathrm{O}}$ increased gradually from $200 \mu \mathrm{L} \cdot \mathrm{min}^{-1}$ to $1000 \mu \mathrm{L} \cdot \mathrm{min}^{-1}$ (i.e. SR 1 to 5). Figure 7 shows the trend observed for the evolution of the $\mathrm{R}_{\mathrm{H}}$ and the PDI when this set of experiments was repeated 3 times (DLS data are shown in Table 3 and in Table S2). For both bioconjugates, XOS- $b$-MeO1 and XOS- $b$ MeRic, a similar trend was observed: when increasing the flow rate $\mathrm{D}_{\mathrm{H} 2 \mathrm{O}}$, the $\mathrm{R}_{\mathrm{H}}$ of the nanoobjects decreased first drastically from $200 \mu \mathrm{L} \cdot \mathrm{min}^{-1}$ to $400 \mu \mathrm{L} \cdot \mathrm{min}^{-1}$, then slowly from 400 
$\mu \mathrm{L} \cdot \mathrm{min}^{-1}$ to $1000 \mu \mathrm{L} \cdot \mathrm{min}^{-1}$ while the PDI slightly increased. The repeatability of these experiments proved that the size of the nano-objects formed could be fine-tuned in these conditions. To further study the morphology of the nano-objects formed, THF was removed under vacuum. The characteristics of the resulting nanoparticles are summarized in Table 3. One can note that the size of nanoparticles obtained at high THF content significantly increased after THF removal, probably because the self-assemblies are very dynamic under these conditions. For higher water content in the final solvent mixture, $\mathrm{R}_{\mathrm{H}}$ measured after THF evaporation are relatively similar than before. In that case, the nano-particles were rapidly kinetically frozen, in agreement with previous experiments $[47,48,51]$.

Finally, the most relevant samples were analyzed by multi-angle light scattering after THF evaporation: sample XOS- $b$-MeOl with SR $1 \& 5$ and sample XOS-b-MeRic with SR $1 \& 5$ (see entries 1, 5, $6 \& 10$ in Table 3), corresponding to the lowest ratio $\mathrm{D}_{\mathrm{THF}} / \mathrm{D}_{\mathrm{H} 2 \mathrm{O}}=200 / 200$ and the highest ratio $\mathrm{D}_{\mathrm{THF}} / \mathrm{D}_{\mathrm{H} 2 \mathrm{O}}=200 / 1000$ giving respectively the largest and the smallest nano-objects. Previously determined sizes were confirmed and the ratio $\mathrm{R}_{\mathrm{g}} / \mathrm{R}_{\mathrm{H}}$ close to $\approx 1$ again confirmed the presence of vesicular morphologies for all these samples.

\section{3- Loading experiments and preliminary biological evaluation}

Nile red loading. The nile red hydrophobic fluorescent dye was co-solubilized firstly in acetone at a concentration of $10^{-4} \mathrm{M}$, then with the THF containing XOS- $b-\mathrm{MeO}$ prior to self-assembly in microfluidics. The mixing with water was performed using a mixing ratio 200-600 (using a flow rate of $200 \mu \mathrm{L} \cdot \mathrm{min}^{-1}$ of THF/acetone containing bioconjugate and nile red mixed with a flow rate of $600 \mu \mathrm{L} \cdot \mathrm{min}^{-1}$ of filtered deionized water) in order to obtain nano-objects not in a metastable state with kinetics of self-assembly relatively fast. The hydrophobic dye should be loaded in the hydrophobic membrane of the vesicles, as confirmed by confocal microscopy observations in Figure S8 (see also video in Supporting Information). Of course, the resolution of the confocal microscope is too low to detect the dye in the membrane but it can be clearly seen that the repartition of the dye is homogeneous through the sample and that it is trapped within small nano-objects with low PDI. This loading experiment was conducted as a proof of concept to show that hydrophobic dye could be encapsulated inside le membrane of the vesicles.

Propiconazole loading and antifungic biological evaluation. Propiconazole is a fungicide used in agriculture, well-known to inhibit the growth of fungi such as trametes versicolor [44]. Five different samples were tested in this study (see Figure 7, D-H) when 
propiconazole was loaded in XOS- $b$-MeOl nano-objects by microfluidics at different concentrations, in comparison with three controls (see Figure 7, A-C). These controls correspond to the trametes versicolor fungi growth in presence of pure water (control 1), XOS- $b-\mathrm{MeOl}$ nano-objects (control 2) and with XOS- $b-\mathrm{MeOl}$ nano-objects and [propiconazole] $=33.7 \mathrm{nmol} . \mathrm{mL}^{-1}$ added on this solution (control 3). All three controls were shown negative and full fungi growth was observed after 17 days (see Figure 7, A-C), even if kinetics studied made on control 1 and 3 showed that full fungi growth was achieved as fast as 12 days (see Figure 8). Figures 7 and 8 show that control 3, where self-assembled nanoparticles were not loaded with propiconazole, were less efficient than the loaded nanoparticles with different amount of propiconazole. Interestingly, fungal growth was almost fully inhibited in all cases when propiconazole was loaded in XOS- $b$-MeOl nanoparticles by microfluidics (see Figure 7, D-H) at least up to 17 days. A concentration-dependence growth inhibition was observed, and interestingly, a significant growth inhibition could be maintained for propiconazole concentration as low as $8.4 \mathrm{nmol} . \mathrm{mL}^{-1}$. Kinetics studies presented in Figure 10 showed that after 13 days, full inhibition of fungal growth was observed for propiconazole loaded in XOS- $b$-MeOl nano-objects at 33.7 and $42.1 \mathrm{nmol} . \mathrm{mL}^{-1}$ where control 1 and 3 presented at this stage full growth of the fungi. The active agent rate release is not constant as full inhibition occurs until 12 days and then slowly decreases linearly from 13 days: as shown in Figure 8, there is full inhibition up to about 12 days at two concentrations of fungicide. By 14 days fungal growth occurs and slowly increases to 27 days. The experiment was carried out until 27 days where the two propiconazole loaded in XOS- $b$-MeOl sample exhibit both an inhibition of fungi superior to $60 \% .100 \%$ growth was achieved when the fungus has occupying the whole agar plate (i.e. $100 \%$ of its surface. Similarly, the percentage of inhibition was calculated depending on the surface of the agar plate not covered by the fungus.

\section{4- Conclusions}

This work led to the synthesis of bio-based amphiphilic oligomers starting from xylans, which are potential byproducts in the paper/pulp industry. After acidic hydrolysis of the xylans to generate well-defined oligomers, the aldehyde function of the reducing end of these oligomers of xylan were then functionalized with an azide group to allow their coupling to fatty acid 
derivatives by 'click chemistry'. Fully bio-sourced bioconjugates were then produced and their self-assembly behavior was studied using three different methods.

The direct solubilization of XOS- $b-\mathrm{MeOl}$ and XOS- $b$-MeRic bioconjugates formed vesicular morphology in water, as evidenced by a combination of TEM, cryo-TEM and light scattering experiments, with a radius around $90 \mathrm{~nm}$ and a relatively high polydispersity. The main advantage of the direct solubilization is the easiness of the preparation that neither require the use of another solvent nor a complex experimental set up. Nevertheless, such a simple approach is not suitable for efficient loading of active materials.

Alternatively, the film-rehydration followed by extrusion method was performed by using THF to form the initial multilamellar film of bioconjugates. As judged by multi-angle DLS combined with SLS, nano-size vesicles were obtained, as confirmed by cryo-TEM. This method has the potentiality to load drugs in the nano-objects formed. Depending on their polarity, different active ingredients can be incorporated in the process by dissolution in the organic solvent for hydrophobic molecules or dissolution in the water for hydrophilic ones. Nile red was loaded in the membrane of the vesicle using this technique by simply adding the fluorescent dye in the THF/bioconjugate mixture used to prepare the starting polymer film.

Finally, a microfluidic set up using a micromixing chip was used to self-assemble bioconjugates in a controlled and reproducible nanoprecipitation approach. Again, vesicles were formed, with a low polydispersity and high reproducibility, as demonstrated by scattering and TEM techniques. In using a third pump for the mixing in the chip or in dissolving a dye/drug in the organic solvent or in the water phase, loading of both hydrophilic and hydrophobic drugs could be easily achieved. This has been first demonstrated by loading nile red, a hydrophobic dye, previously dissolved in the THF containing bioconjugates. Then propiconazole, a potent anti-fungic molecule was loaded at different concentrations. The evaluation of the anti-fungic activity of propiconazole loaded into our bioconjugate XOS- $b$ $\mathrm{MeOl}$ vesicles was then compared to free propiconazole and evidenced the enhanced activity of the drug encapsulated by reducing significantly the growth of trametes versicolor (i.e selfassembled nanoparticles not loaded with propiconazole were less efficient in slow delivery than the loaded nanoparticles with different amount of propiconazole).

\section{Conflicts of interest}

There are no conflicts to declare. 


\section{Acknowledgements}

This work is financially supported by the Aquitaine Council, FCBA, ITERG and ANR-10EQPX-16-XYLOFOREST. The authors thank P. Castel (CESAMO, Institut des Sciences Moléculaires, Université de Bordeaux, Talence (33), France) for MALDI-TOF MS experiments. The transmission electron microscopy (TEM) was done in the Bordeaux Imaging Center, a service unit of the CNRS-INSERM and Bordeaux University, member of the national infrastructure France BioImaging, so the authors thank S. Lacomme for the training. E. Ibarboure (Laboratoire de Chimie des Polymères Organiques, LCPO, Université de Bordeaux, Pessac (33607), France) is acknowledged for confocal microscopy measurements. Finally, M. Coloignier (ITERG, 11 rue Gaspard Monge, Parc Industriel, Pessac, (33600), France) is thanked for advices in the XOS hydrolysis scale-up and fruitful discussions.

\section{Notes and references}

[1] Ramesh, H. P.; Tharanatan, N. T. Carbohydrates-The Renewable Raw Materials of High Biotechnological Value. Critical Reviews in Biotechnology 2003, 23 (2), 149-173.

[2] Girio, F.M.; Fonseca, C.; Carvalheiro, F.; Duarte, L. C.; Marques, S.; Bogel-Lukasik, R. Hemicelluloses for Fuel Ethanol: a Review. Bioresource Technology. 2010, 101 (13), 4775-4800.

[3] Ragauskas, A. J.; Williams, C. K.; Davison, B. H.; Britovsek, G.; Cairney, J.; Eckert, C. A.; Frederick Jr., W. J.; Hallett, J. P.; Leak, D. J.; Liotta, C. L.; Mielenz, J. R.; Murphy, R.; Templer, R.; Tschaplinski, T. The Path Forward for Biofuels and Biomaterials. Science. 2006, 311 (5760), 484-489.

[4] Deutschmann, R.; Dekker, R. F. H. From Plant Biomass to Bio-Based Chemicals: Latest Developments in Xylan Research. Biotechnology Advances. 2012, 30 (6), 1627-1640.

[5] Izydorczyk, M. S.; Biliaderis, C. G. Cereal Arabinoxylans: Advances in Structure and Physicochemical Properties. Carbohydrate Polymers. 1995, 28 (1), 33-48.

[6] Ebringerova, A.; Hromadkova. Z. Xylans of Industrial and Biomedical Importance. Biotechnol. Genet. Eng. Rev. 1999, 16, 325-346.

[7] Ebringerova, A.; Heinze, T. Xylan and Xylan Derivatives - Biopolymers with Valuable Properties, 1. Naturally Occurring Xylans Structures, Isolation Procedures and Properties. Macromol. Rapid Commun. 2000, 21 (9), 542-556. 
[8] Mäki-Arvela, P.; Salmi, T.; Holmbom, B.; Willfor, S.; Murzin, D. Yu. Synthesis of Sugars by Hydrolysis of Hemicelluloses- A Review. Chem. Rev. 2011, 111 (9), 5638-5666.

[9] Sun, H. J.; Yoshida, S.; Park, N. H.; Kusakabe, I. Preparation of $(1 \rightarrow 4)-\beta$-dXylooligosaccharides from an Acid Hydrolysate of Cotton-Seed Xylan: Suitability of CottonSeed Xylan as a Starting Material for the Preparation of $(1 \rightarrow 4)-\beta-\mathrm{d}$-Xylooligosaccharides. Carbohydrate Research. 2002, 337 (7), 657-661.

[10] Blanazs, A.; Armes, S. P.; Ryan, A. J. Self-Assembled Block Copolymer Aggregates: from Micelles to Vesicles and their Biological Applications. Macromol. Rapid Commun. 2009, 30 (4-5), 267-277.

[11] Schatz, C.; Lecommandoux, S. Polysaccharide-Containing Block Copolymers: Synthesis, Properties and Applications of an Emerging Family of Glycoconjugates. Macromol. Rapid Commun. 2010, 31 (19), 1664-1684.

[12] Ziegast, G.; Pfannemüller, B. Linear and Star-Shaped Hybrid Polymers. Part IV. Phosphorolytic Syntheses with Di-, Oligo- and Multifunctional Primers. Carbohydrate Research. 1987, 160, 185-204.

[13] Yagi, S.; Kasuya, N.; Fukuda, K. Synthesis and Characterization of Cellulose- $b$ Polystyrene. Polym. J. 2010, 42 (4), 342-348.

[14] Tornoe, C. W.; Christensen, C.; Meldal, M. Peptidotriazoles on Solid Phase: [1,2,3]Triazoles by Regiospecific Copper(I)-Catalyzed 1,3-Dipolar Cycloadditions of Terminal Alkynes to Azides. J. Org. Chem. 2002, 67 (9), 3057-3064.

[15] Bernard, J.; Save, M.; Arathoon, B.; Charleux, B. Preparation of a xanthate-terminated dextran by click chemistry: Application to the synthesis of polysaccharide-coated nanoparticles via surfactant-free ab initio emulsion polymerization of vinyl acetate. J. Polym. Sci., Part A: Polym. Chem. 2008, 46 (8), 2845-2857.

[16] Li, B. G.; Zhang, L. M. Synthesis and characterization of novel amphiphilic block copolymers based on maltoheptaose and poly(E-caprolactone). Carbohydrate Polymers. 2008, 74 (3), 390-395.

[17] Lohmann, J.; Houga, C.; Driguez, H.; Wilson, J.; Destarac, M.; Fort, S.; Le Meins, J.F.; Borsali, R.; Taton, D.; Gnanou, Y. Hybrid Block Copolymers Incorporating Oligosasaccharides and D Synthetic Blocks Grown by Controlled Radical Polymerization. ACS Symp. Ser. 2009, 1023, 231-240.

[18] Upadhyay, K. K.; Le Meins, J.-F.; Misra, A.; Voisin, P.; Bouchaud, V.; Ibarboure, E.; Schatz, C.; Lecommandoux, S. Biomimetic Doxorubicin Loaded Polymersomes from Hyaluronan-block-Poly( $\gamma$-benzyl glutamate) Copolymers. Biomacromolecules. 2009, 10 (10), 2802-2808. 
[19] Bonduelle, C.; Oliveira, H.; Gauche, C.; Huang, J.; Heise, A.; Lecommandoux, S. Multivalent Effect of Glycopolypeptide Based Nanoparticles for Galectin Binding. Chem. Commun. 2016, 52 (75), 11251-11254.

[20] Chemin, M.; Rakotovelo, A.; Ham-Pichavant, F.; Chollet, G.; da Silva Perez, D.; PetitConil, M.; Cramail, H.; Grelier. S. Synthesis and characterization of functionalized 4-Omethylglucuronoxylan derivatives. Holzforschung. 2015, 69 (6), 713-720.

[21] Chemin, M.; Wirotius, A-L.; Ham-Pichavant, F.; Chollet, G.; Da Silva Perez, D.; Petit-Conil, M.; Cramail, H.; Grelier. S. Well-defined oligosaccharides by mild acidic hydrolysis of hemicelluloses. European Polymer Journal. 2015, 66, 190-197.

[22] C. Schatz, C.; Louguet, S.; Le Meins, J-F.; Lecommandoux, S. Polysaccharide-block-polypeptide Copolymer Vesicles: Towards Synthetic Viral Capsids. Angew. Chemie Int. Ed., 2009, 48 (14), 2572-2575.

[23] Huynh, N-T.; Jeon, Y-S.; Zrinyi, M.; Kim. J-H. Preparation of 'click' hydrogels from polyaspartamide derivatives. Polym Int. 2013, 62 (2), 266-272.

[24] Anastasaki, A.; Haddleton, A. J.; Zhang, Q.; Simula, A.; Droesbeke, M.; Wilson, P.; Haddleton, D. M. Aqueous Copper-Mediated Living Radical Polymerisation of NAcryloylmorpholine, SET-LRP in Water. Macromol. Rapid. Commun. 2014, 35 (10), 965970.

[25] Diaz, D. D.; Punna, S.; Holzer, P.; McPherson, A. K.; Sharpless, K. B.; Fokin, V. V.; Finn, M. G. J. Click Chemistry in Materials synthesis. 1. Adhesive Polymers from Copper-Catalyzed Azide-Alkyne Cycloaddition. Polym. Sci., Part A: Polym. Chem. 2004, 42 (17), 4392-4403.

[26] de Espinosa, L.; Meier, M. A. R. Synthesis of Star- and Block-Copolymers using ADMET: Head-to-Tail Selectivity During Step-Growth Polymerization. Chem. Commun. 2011, 47 (6), 1908-1910.

[27] Meier, M. A. R.; Metzger, J. O.; Schubert, U. S. Plant Oil Renewable Resources as Green Alternatives in Polymer Science. Chem. Soc. Rev. 2007, 36 (11), 1788-1802.

[28] More, A. S.; Maisonneuve, L.; Lebarté, T.; Gadenne, B.; Alfos, C.; Cramail, H. Vegetable-Based Building-Blocks for the Synthesis of Thermoplastic Renewable Polyurethanes and Polyesters. Eur. J. Lipid Sci. Technol. 2013, 115 (1), 61-75.

[29] Petrovic, Z. S. Polyurethanes from Vegetable Oils. Polym. Rev. 2008, 48 (1), 109-155.

[30] Hong, J.; Luo, Q.; Wan, X.; Petrovic, Z. S.; Shah, B. K. Biopolymers from Vegetable Oils via Catalyst- and Solvent-Free "Click" Chemistry: Effects of Cross-Linking Density. Biomacromolecule. 2012, 13 (1), 261-266.

[31] Ogunniyi, D. S. Castor Oil: A Vital Industrial Raw Material. Bioresour. Technol. 2006, 97 (9), 1086-1091. 
[32] Maisonneuve, L.; Lebarbe, T.; Grau, E.; Cramail, H. Structure-Properties Relationship of Fatty Acid-Based Thermoplastics as Synthetic Polymer Mimics. Polym. Chem. 2013, 4 (22), 5472-5517.

[33] Biermann, U.; Bornscheuer, U.; Meier, M. A. R.; Metzger, J. O.; Schäfer, H. J. Oils and Fats as Renewable Raw Materials in Chemistry. Angew, Chem., Int. Ed., 2011, 50 (17), 3854-3871.

[34] Jérôme, F.; Kharchafi, G.; Adam, I.; Barrault, J. "One Pot" and Selective Synthesis of Monoglycerides over Homogeneous and Heterogeneous Guanidine Catalysts. Green Chem. 2004, 6 (2), 72-74.

[35] Jérôme, F.; Kharchafi, G.; Adam, I.; Barrault, J. "One Pot" and Selective Synthesis of Monoglycerides over Homogeneous and Heterogeneous Guanidine Catalysts. Green Chem. 2004, 6 (2), 72-74.

[36] Lebarbé, T.; Maisonneuve, L.; Nga Nguyen, T. H.; Gadenne, B.; Alfos, C.; Cramail, H. Methyl 10-Undecenoate as a Raw Material for the Synthesis of Renewable SemiCrystalline Polyesters and Poly(ester-amide)s. Polym. Chem. 2012, 3 (10), 2842-2851.

[37] Kolb, H. C.; Finn, M. G.; Sharpless, K. B. Click Chemistry: Diverse Chemical Function from a Few Good Reactions. Angew, Chem., Int. Ed. 2001, 40 (11), 2011-2021.

[38] Lligadas, G.; Ronda, J. C.; Galià, M.; Cádiz, V. Monomers and Polymers from Plant Oils via Click Chemistry Reactions J. Polym. Sci., Part A: Polym. Chem. 2013, 51 (10), 21112124.

[39] Upadhyay, K. K.; Le Meins, J. F.; Misra, A.; Voisin, P.; Bouchaud, V.; Ibarboure, E.; Schatz, C.; Lecommandoux, S. Biomimetic Doxorubicin Loaded Polymersomes from Hyaluronan-block-Poly( $\gamma$-benzyl glutamate) Copolymers. Biomacromolecules. 2009, 10 (10), 2802-2808.

[40] Chécot, F.; Lecommandoux, S.; Klok, H-A.; Gnanou, Y. From Supramolecular Polymersomes to Stimuli-Responsive Nano-Capsules Based on Poly(diene- $b$-peptide) Diblock Copolymers. Eur. Phys. J. E. 2003, 10 (1), 25-35.

[41] Kratochvil, P. Classical Light Scattering from Polymer Solutions, edited by A.D. Jenkins (Elsevier, Amsterdam, 1987).

[42] Benoit, H.; Froehlich, D. Light Scattering from Polymer Solutions, edited by M.B. Huglin (Academic Press, London, 1972).

[43] Samad, A.; Sultana, Y.; Aqil, M. Liposomal Drug Delivery Systems: an Update Review. Current Drug Delivery, 2007, 4 (4), 297-305.

[44] Bleul, R.; Thiermann, R.; Maskos, M. Techniques to Control Polymersome Size. Macromolecules, 2015, 48 (20), 7396-7409. 
[45] Shum, H. C.; Kim, J.-W.; Weitz, D. A. Microfluidic Fabrication of Monodisperse Biocompatible and Biodegradable Polymersomes with Controlled Permeability. J. Am. Chem. Soc., 2008, 130 (29), 9543-9549.

[46] Habault, D.; Dery, A.; Leng, J.; Lecommandoux, S.; Le Meins, J. F.; Sandre, O. Droplet Microfluidics to Prepare Magnetic Polymer Vesicles and to Confine the Heat in Magnetic Hyperthermia. IEEE Trans. Magn. 2013, 49 (1), 182-190.

[47] Johnson, B. K.; Prud'homme, R. K. Mechanism for Rapid Self-Assembly of Block Copolymer Nanoparticles. Phys. Rev. Lett. 2003, 91 (11), 118302/1-118302/1.

[48] Saad, W. S.; Prud'homme, R. K. Principles of Nanoparticle Formation by Flash Nanoprecipitation. Nanotoday. 2016, 11 (2), 212-227.

[49] Thiermann, R.; Mueller, W.; Montesinos-Castellanos, A.; Metzke, D.; Löb, P.; Hessel, V.; Maskos, M. Size Controlled Polymersomes by Continuous Self-Assembly in Micromixers. Polymer. 2012, 53 (11), 2205-2210.

[50] Bleul, R.; Thiermann, R.; Marten, G. U.; House, M. J.; St. Pierre, T. G.; Häfeli, U. O.; Maskos, M. Continuously Manufactured Magnetic Polymersomes - a Versatile Tool (not only) for Targeted Cancer Therapy. Nanoscale. 2013, 5 (23), 11385-1193.

[51] Le Fer, G.; Portes, D.; Goudounet, G.; Guigner, J. M.; Garanger, E.; Lecommandoux. S. Design and Self-Assembly of PBLG-b-ELP Hybrid Diblock Copolymers Based on Synthetic and Elastin-Like Polypeptides. Org. Biomol. Chem. 2017, 15 (47), 10095-10104.

[52] Lekounougou, S.; Pétrissans, M.; Jacquot, L. P.; Gelhaye, E.; Gérardin, P. Effect of Heat Treatment on Extracellular Enzymatic Activities Involved in Beech Wood Degradation by Trametes Versicolor. Wood Science and Technology. 2009, 43 (3-4), 331-341. 


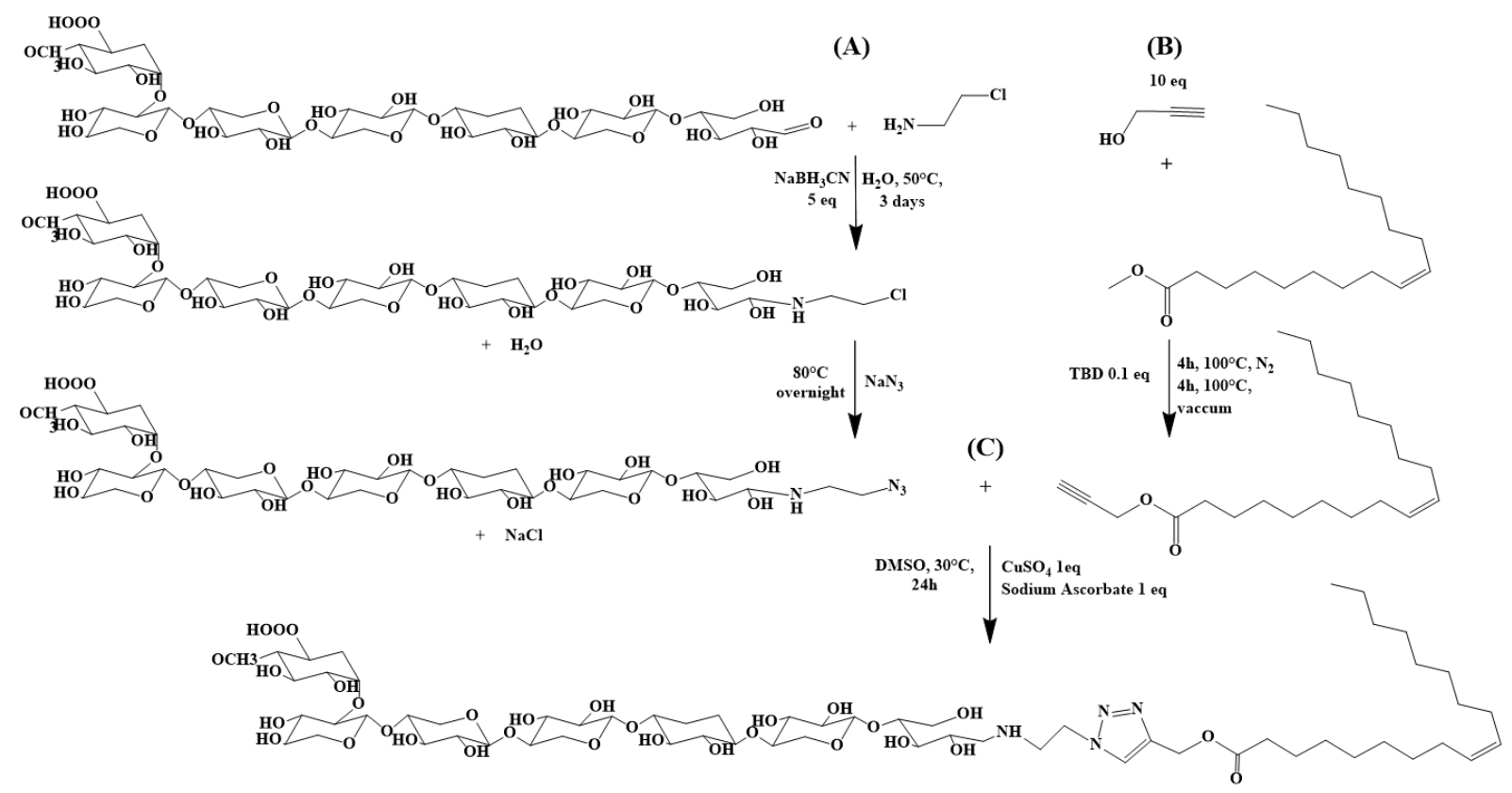

Scheme 1 Overall reactions Scheme illustrating the formation of the fully bio-sourced amphiphilic bioconjugates. (A) reductive amination of the xylo-oligosaccharide with 2aminoethyl chloride followed by reaction with sodium azide, (B) transesterification reaction with propargyl alcohol illustrated on methyl oleate (MeOl) (C) Huisgen cycloaddition illustrated between the azide functionalized XOS and the alkyne functionalized methyl oleate. 


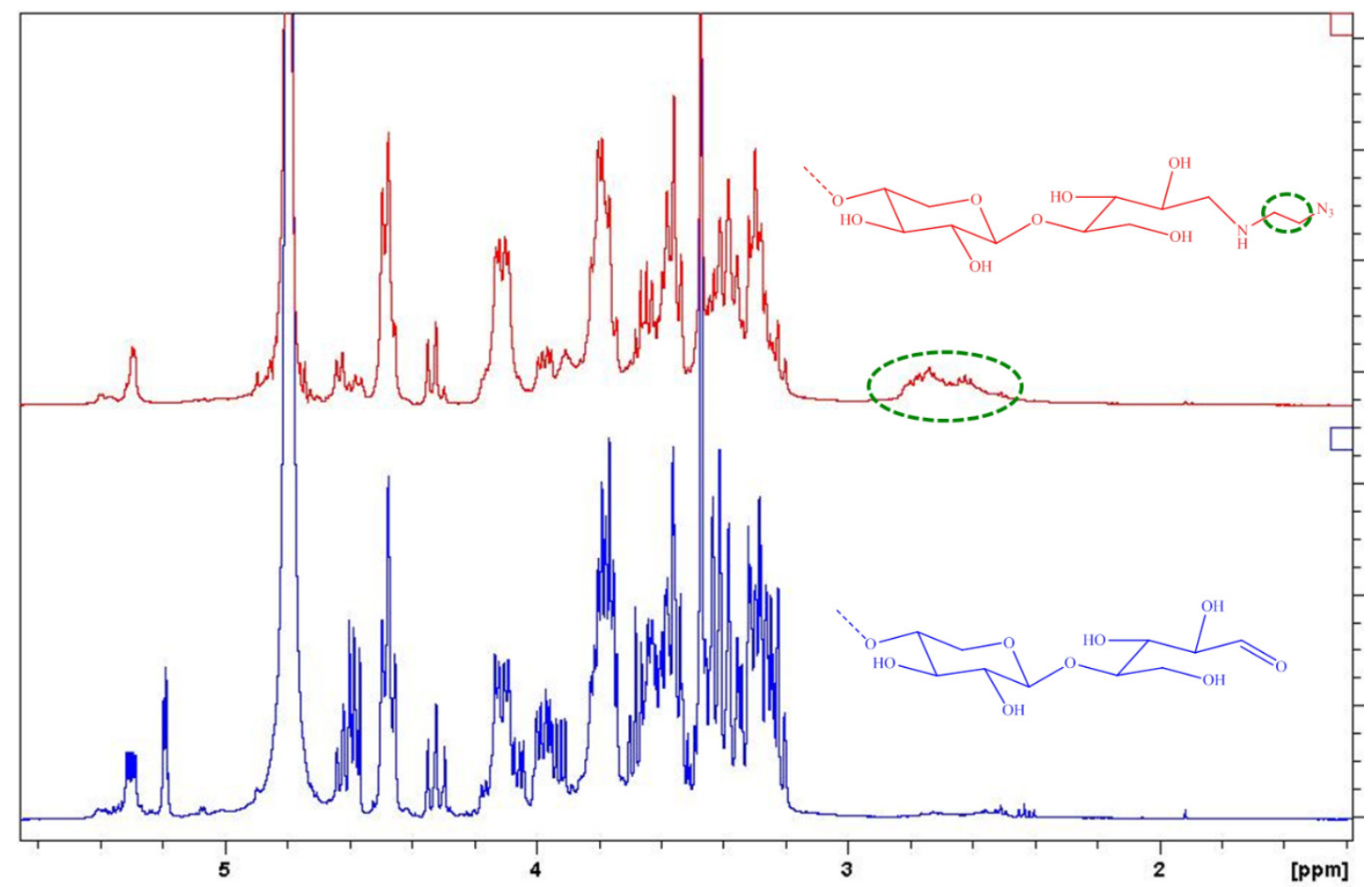

Fig. $1{ }^{1} \mathrm{H}$ NMR spectra in $\mathrm{D}_{2} \mathrm{O}$ of xylo-oligosaccharides before (blue) and after (red) reductive amination with 2 -aminoethyl chloride followed by reaction with sodium azide. 

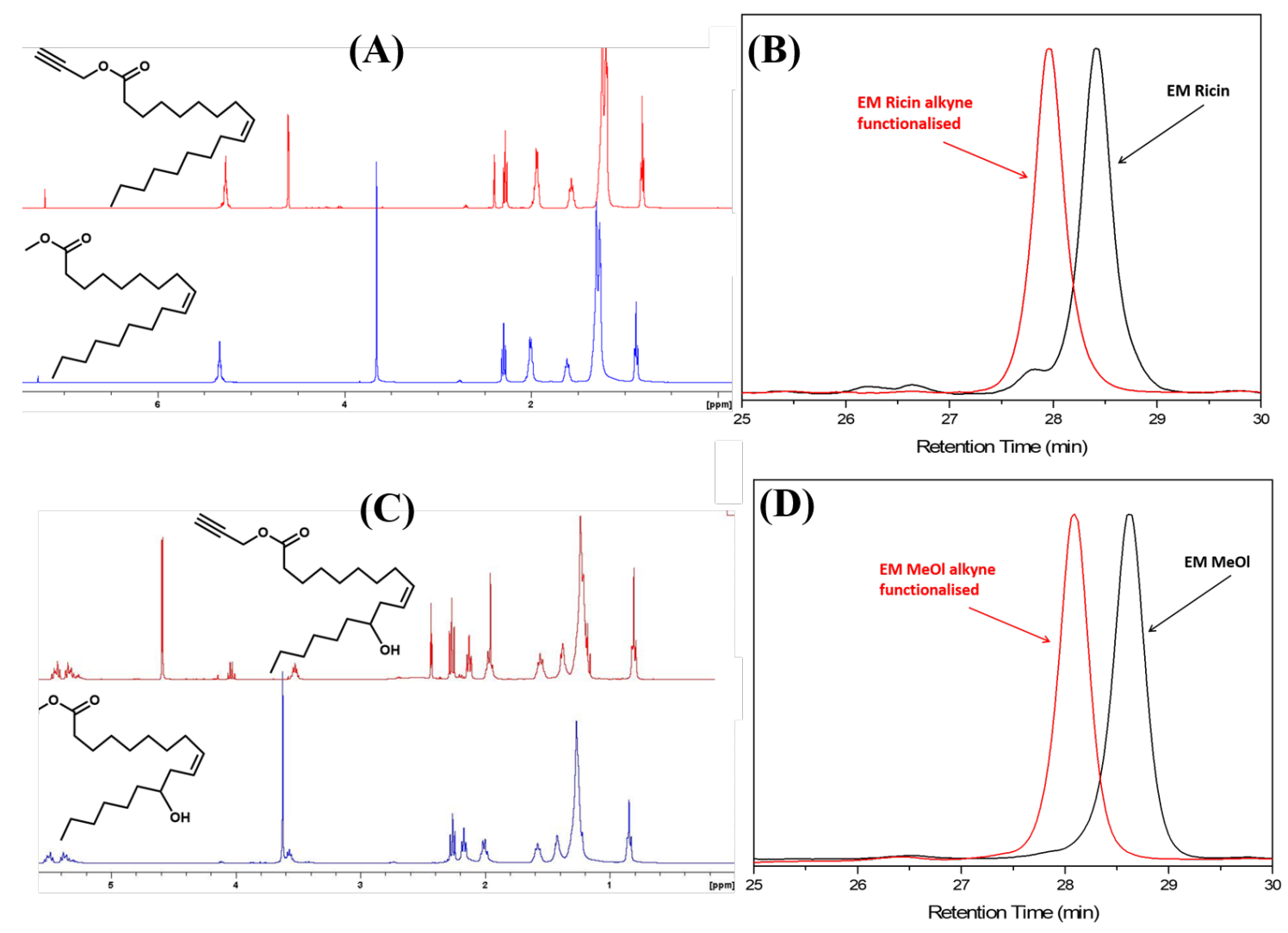

Fig. 2 Fatty Acid Methyl Ester transesterification with propargyl alcohol. ${ }^{1} \mathrm{H}$ NMR spectra in $\mathrm{CDCl}_{3}$ of the methyl oleate (A) before (blue) and after (red) transesterification with propargyl alcohol, of the methyl ricinoleate (C) before (blue) and after (red) transesterification with propargyl alcohol; GPC traces in THF of the methyl oleate (B) before (black) and after (red) transesterification with propargyl alcohol and of the methyl ricinoleate (D) before (black) and after (red) transesterification with propargyl alcohol using a RI detector calibrated with PMMA. 


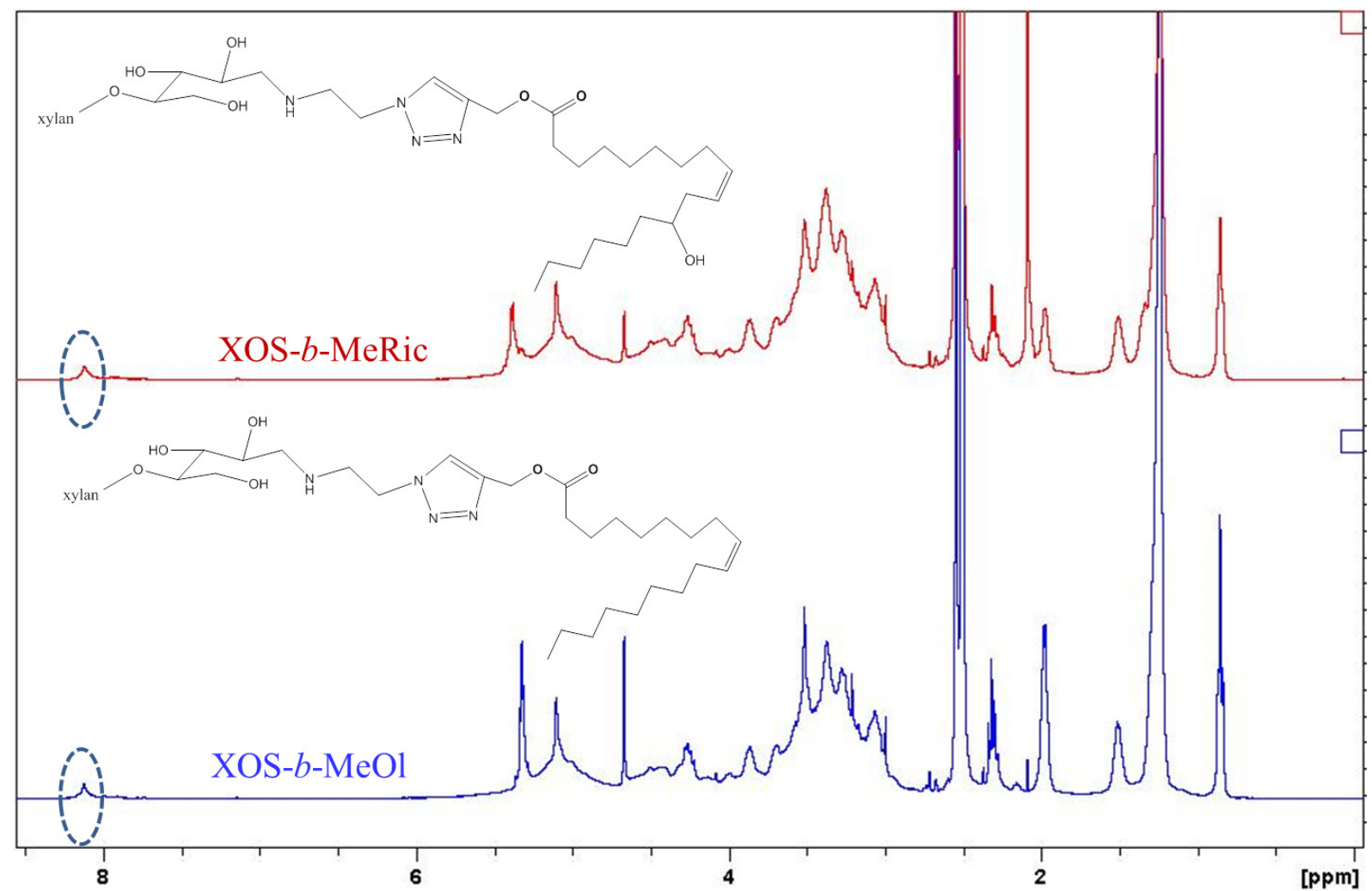

Fig. 3 Overlaid spectra of the ${ }^{1} \mathrm{H}$ NMR of the bioconjugates XOS- $b$-MeOl (bottom) and XOS$b$-MeRic (top) in DMSO-d6. 

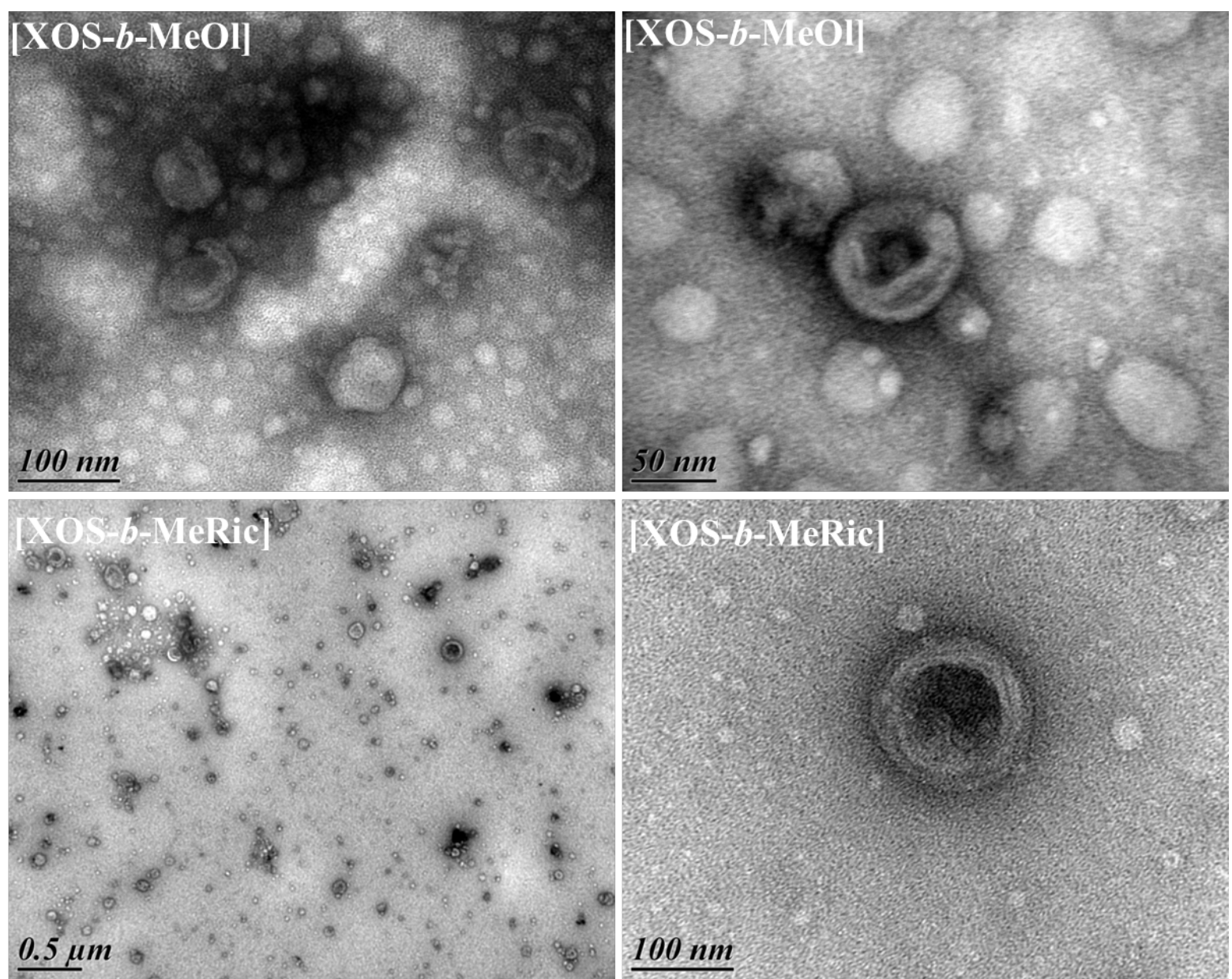

Fig. 4 TEM images obtained for the bioconjugates XOS- $b-\mathrm{MeOl}$ and XOS- $b$-MeRic after direct solubilization in $\mathrm{H}_{2} \mathrm{O}\left(1 \mathrm{mg} \cdot \mathrm{mL}^{-1}\right)$ and stained with uranyl formate. 


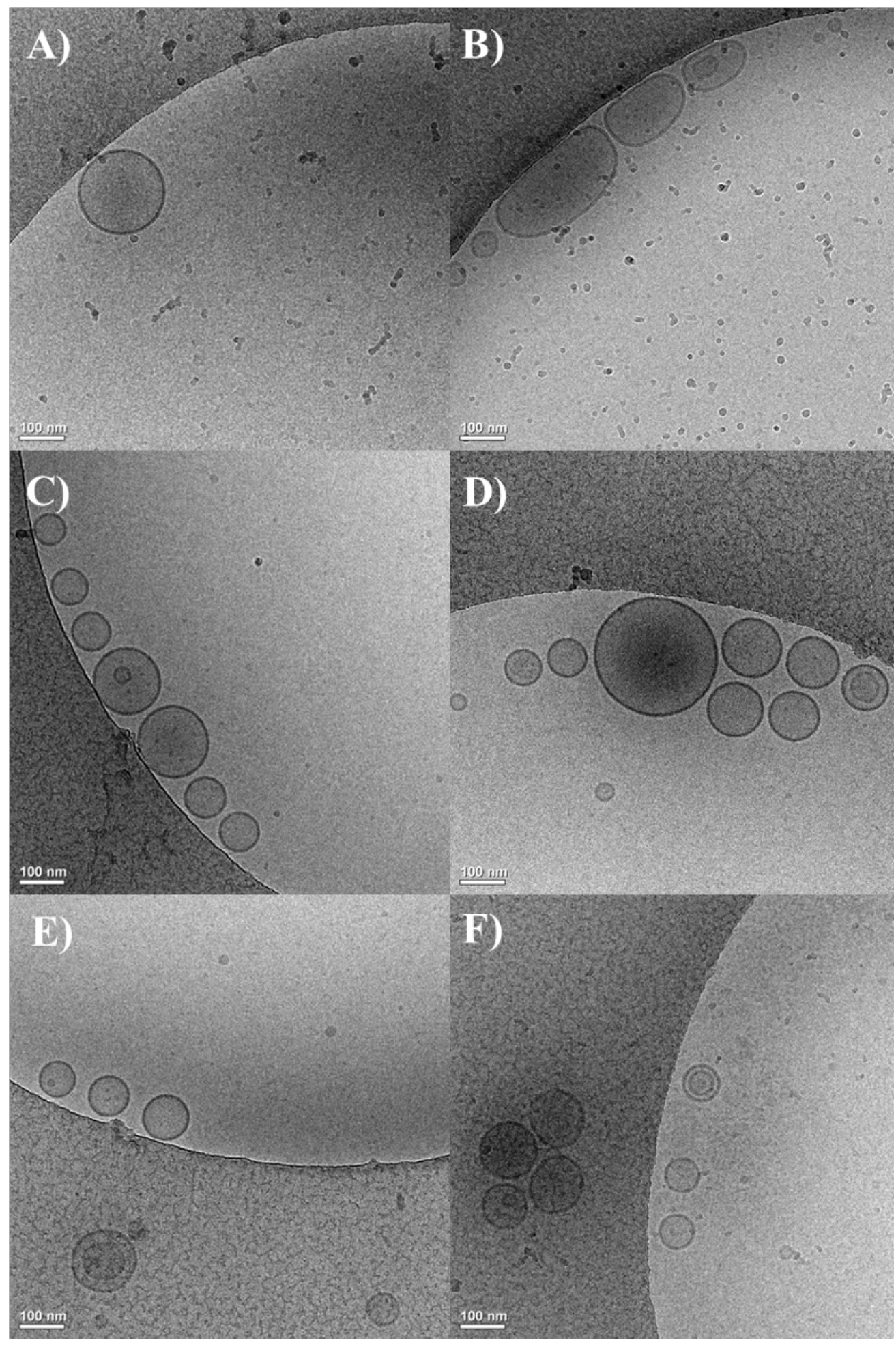

Fig. 5 Cryo-TEM images obtained after direct solubilization in $\mathrm{H}_{2} \mathrm{O}\left(6 \mathrm{mg} \cdot \mathrm{mL}^{-1}\right)$ for the bioconjugates XOS- $b$-MeOl (A, B) and XOS-b-MeRic (C, D) and after thin-film rehydration followed by extrusion for the bioconjugate XOS- $b-\mathrm{MeOl}(\mathrm{E}, \mathrm{F})$. 


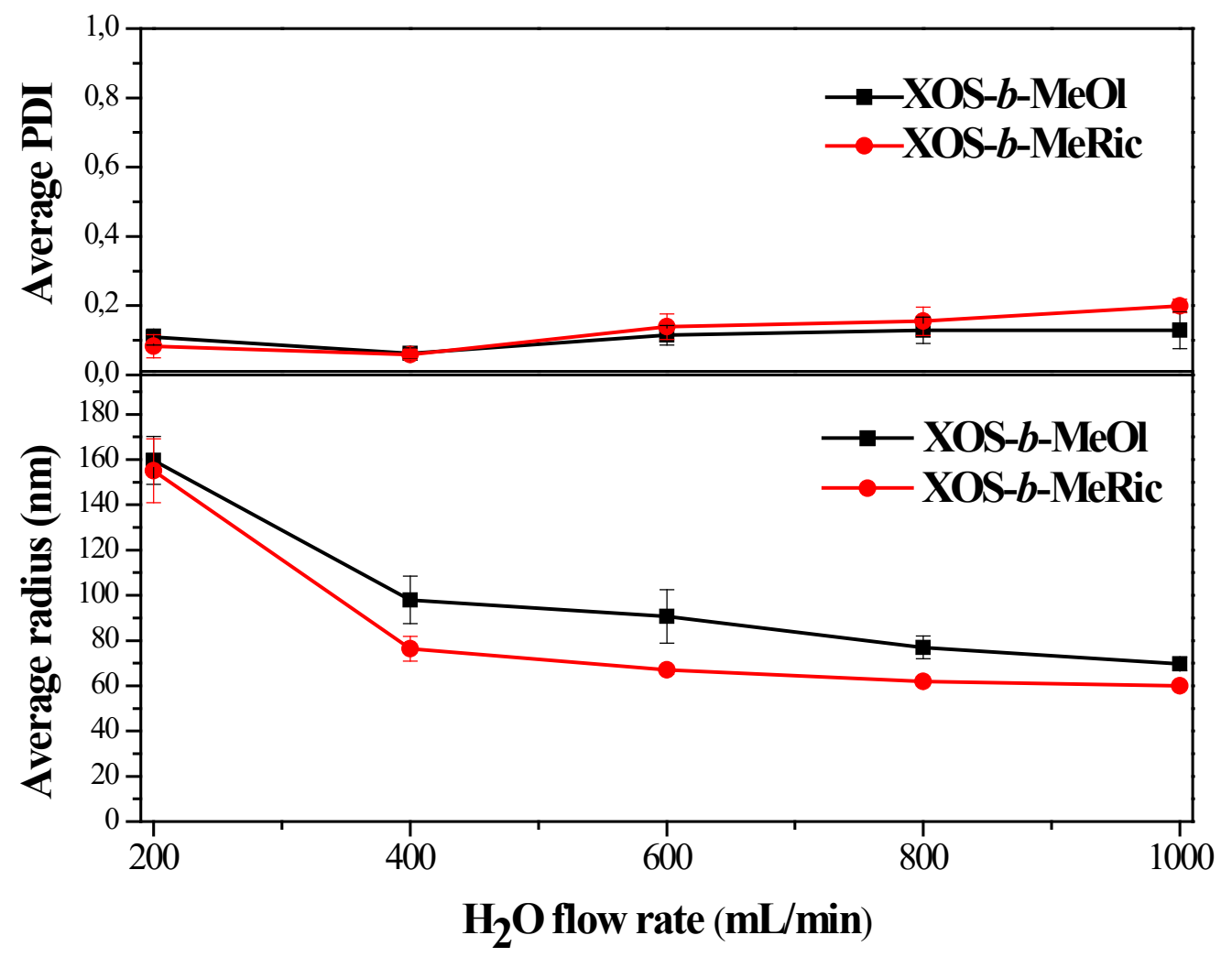

Fig. 6 Evolution of the average radii and polydispersity index obtained by DLS from the amphiphilic bioconjugates XOS- $b$-MeOl and XOS- $b$-MeRic vesicles after self-assembly using a microfluidics system. The bioconjugates were dispersed in THF at a concentration of $2 \mathrm{mg} \cdot \mathrm{mL}^{-1}$ and mixed with increasing flow rates of water. 

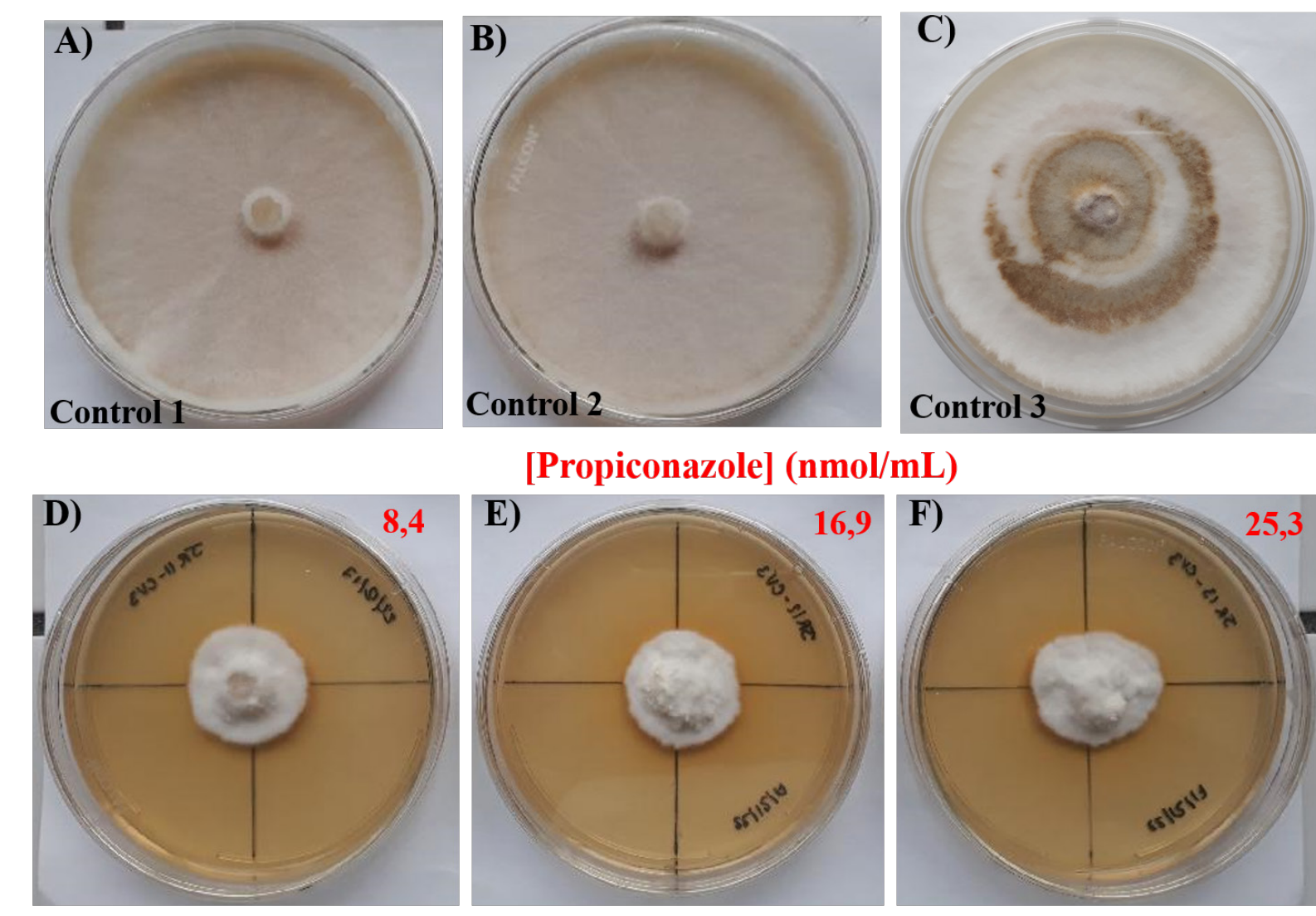

[Propiconazole] (nmol/mL)
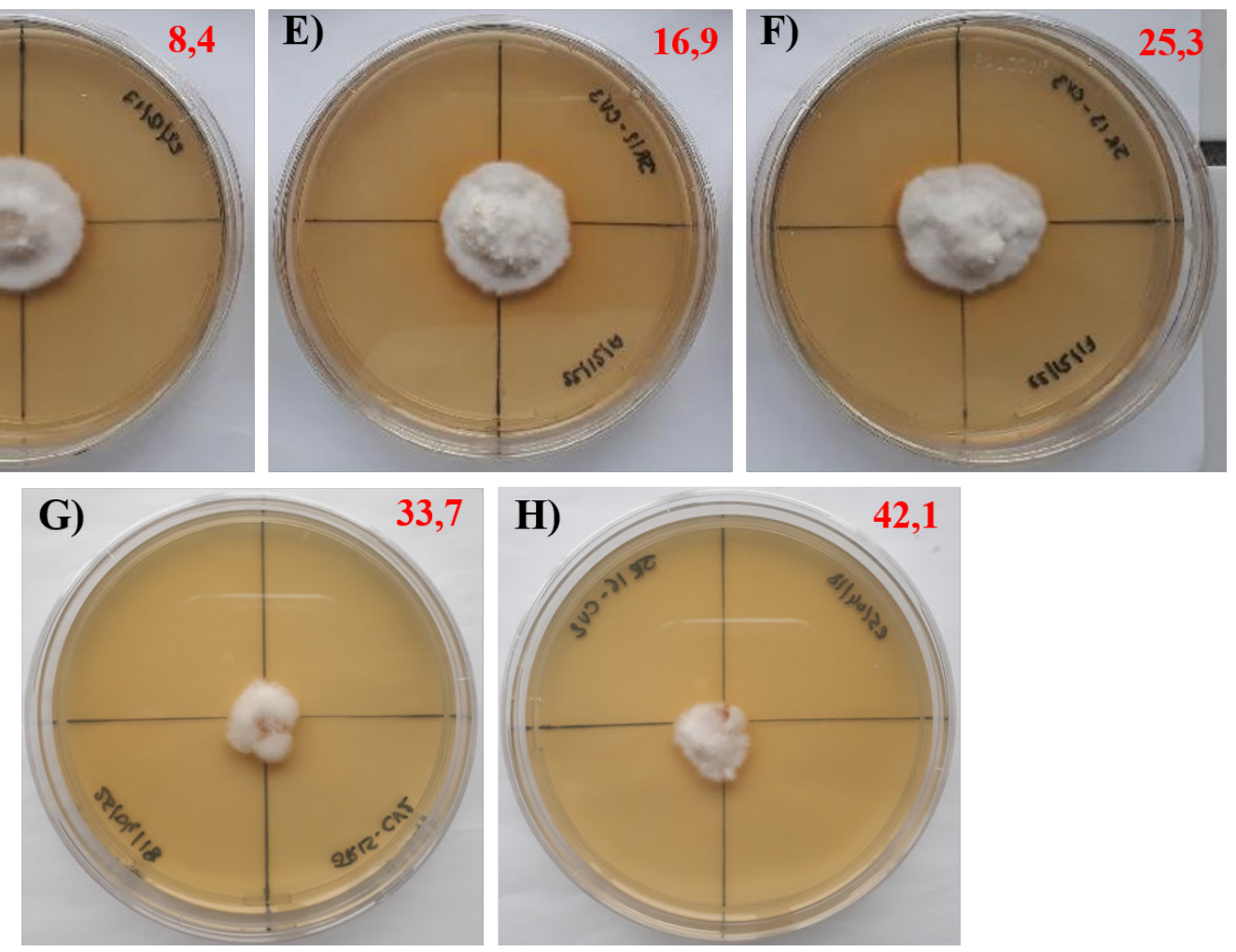

Fig. 7 Picture of the fungal growth after propagation of the strain Trametes versicolor on malt/agar $(4 \% / 2 \%)$ plates incubated at $22^{\circ} \mathrm{C}$ and $70 \%$ of relative humidity for 17 days, B), DF) and for 19 days, A), C), G), H). A) control 1 with water added to the malt/agar medium, B) control 2 with [XOS- $b$-MeOl] self-assembled by microfluidics added to the malt/agar medium, C) control 3 with [XOS- $b$-MeOl] self-assembled by microfluidics followed by addition of [propiconazole] $=33.7 \mathrm{nmol} . \mathrm{mL}^{-1}$ added to the malt/agar medium and D-H) with [XOS- $b-\mathrm{MeOl}$ ] self-assembled by microfluidics with an increasing amount [propiconazole] $=$ (D) 8.4, (E) 16.9, (F) 25.3, (G) 33.7, (H) $42.1 \mathrm{nmol} . \mathrm{mL}^{-1}$ added to the malt/agar medium. 


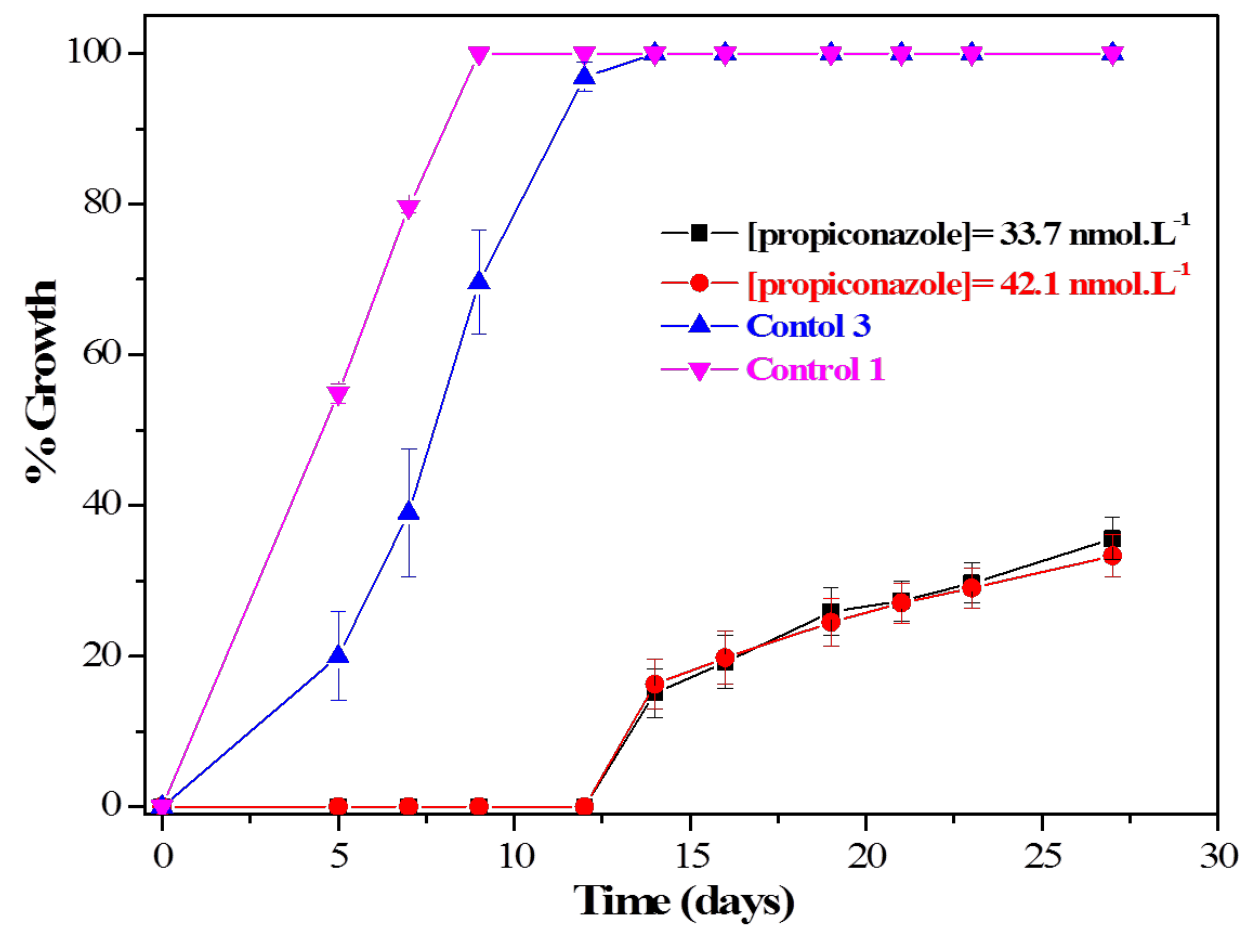

Fig. 8 Kinetic evolution of the fungal growth after propagation of the strain Trametes versicolor on malt/agar $(4 \% / 2 \%)$ plates incubated at $22^{\circ} \mathrm{C}$ and $70 \%$ of relative humidity for control 1 with water added to the malt/agar medium, control 3 with [XOS- $b$-MeOl] selfassembled by microfluidics followed by addition of [propiconazole] $=33.7 \mathrm{nmol} . \mathrm{mL}^{-1}$ added to the malt/agar medium and with [XOS- $b-\mathrm{MeOl}$ ] self-assembled by microfluidics with [propiconazole] $=33.7$ and $42.1 \mathrm{nmol} . \mathrm{mL}^{-1}$ added to the malt/agar medium. 


\begin{tabular}{|c|c|c|c|c|c|c|}
\hline \multicolumn{2}{|c|}{} & \multicolumn{2}{c|}{$\begin{array}{c}\text { Multi-angle } \\
\text { DLS at 90 }\end{array}$} & \multicolumn{2}{c|}{ (Malvern) } & \multicolumn{2}{c|}{$\begin{array}{c}\text { DLS and SLS } \\
\text { (ALV) }\end{array}$} \\
\hline Sample & Solvent & $\begin{array}{c}\text { Concentration } \\
(\mathbf{m g} / \mathbf{m L})\end{array}$ & $\begin{array}{c}\mathbf{R}_{\mathbf{H}} \\
(\mathbf{n m})\end{array}$ & PDI & $\begin{array}{c}\text { Rg } \\
\text { (nm) }\end{array}$ & $\begin{array}{c}\mathbf{R}_{\mathbf{H}} \\
\text { (nm) }\end{array}$ \\
\hline XOS- $b$-MeOl & $\mathrm{H}_{2} \mathrm{O}$ & 1 & 87 & 0.16 & 91 & 104 \\
\hline XOS- $b$-MeRic & $\mathrm{H}_{2} \mathrm{O}$ & 1 & 91 & 0.19 & 66 & 61 \\
\hline XOS- $b$-MeOl & $\mathrm{CHCl}_{3}$ & 10 & 74 & 0.16 & 71 & 34 \\
\hline XOS- $b$-MeRic & $\mathrm{CHCl}_{3}$ & 10 & 89 & 0.12 & 46 & 29 \\
\hline
\end{tabular}

Table 1 Summary of the sizes obtained by DLS at $90^{\circ}$ using Malvern and multi-angle DLS and SLS using the ALV for the samples XOS- $b$-MeOl and XOS- $b$-MeRic by direct solubilization in water and in chloroform.

\begin{tabular}{|c|c|c|c|c|c|c|}
\hline \multicolumn{2}{|c|}{ Thin film rehydration } & \multicolumn{3}{|c|}{ DLS at 90 ${ }^{\circ}$ (Malvern) } & \multicolumn{2}{c|}{$\begin{array}{c}\text { Multi-angle DLS } \\
\text { and SLS (ALV) }\end{array}$} \\
\hline Sample & Extrusion & $\begin{array}{c}\mathbf{R}_{\mathbf{H}} \\
(\mathbf{n m})\end{array}$ & PDI & Comments & $\begin{array}{c}\text { Rg } \\
(\mathbf{n m})\end{array}$ & $\begin{array}{c}\mathbf{R}_{\mathbf{H}} \\
(\mathbf{n m})\end{array}$ \\
\hline XOS- $b$-MeOl & No & 128 & 0.35 & Multimodal & - & - \\
\hline XOS- $b$-MeOl & $0.4 \mu \mathrm{m}$ & 74 & 0.23 & Monomodal & 44 & 51 \\
\hline XOS- $b$-MeRic & No & 189 & 0.38 & Multimodal & - & - \\
\hline XOS- $b$-MeRic & $0.4 \mu \mathrm{m}$ & 86 & 0.28 & Monomodal & 44 & 37 \\
\hline
\end{tabular}

Table 2 Summary of the sizes obtained by DLS at $90^{\circ}$ using Malvern and multi-angle DLS and SLS using the ALV after thin film rehydration and extrusion for the bioconjugates XOS$b$-MeRic and XOS- $b$-MeOl. 


\begin{tabular}{|c|c|c|c|c|c|c|c|c|c|}
\hline \multicolumn{2}{|c|}{ Microfluidic } & \multicolumn{4}{|c|}{ In $\mathrm{THF} / \mathrm{H}_{2} \mathrm{O}$ mixture } & \multicolumn{4}{|c|}{ After THF evaporation } \\
\hline \multirow[b]{2}{*}{ Exp } & \multirow[b]{2}{*}{ Sample } & \multirow[b]{2}{*}{$\mathbf{D}_{\mathrm{H} 20}$} & \multirow[b]{2}{*}{ SR } & \multicolumn{2}{|c|}{$\begin{array}{l}\text { DLS at } 90^{\circ} \\
\text { (Malvern) }\end{array}$} & \multicolumn{2}{|c|}{$\begin{array}{l}\text { DLS at } 90^{\circ} \\
\text { (Malvern) }\end{array}$} & \multicolumn{2}{|c|}{$\begin{array}{c}\text { Multi-angle } \\
\text { DLS and } \\
\text { SLS (ALV) }\end{array}$} \\
\hline & & & & $\begin{array}{c}\mathbf{R}_{\mathrm{H}} \\
(\mathrm{nm})\end{array}$ & PDI & $\begin{array}{c}\mathbf{R}_{\mathrm{H}} \\
(\mathbf{n m})\end{array}$ & PDI & $\begin{array}{c}\text { Rg } \\
(\mathrm{nm})\end{array}$ & $\begin{array}{c}\mathrm{Rh} \\
(\mathrm{nm})\end{array}$ \\
\hline 1 & XOS- $b$-MeOl & 200 & 1 & 175 & 0.14 & 374 & 0.13 & 154 & 177 \\
\hline 2 & XOS- $b-\mathrm{MeOl}$ & 400 & 2 & 115 & 0.07 & 219 & 0.06 & - & - \\
\hline 3 & XOS- $b-\mathrm{MeOl}$ & 600 & 3 & 110 & 0.07 & 173 & 0.10 & - & - \\
\hline 4 & XOS- $b$-MeOl & 800 & 4 & 85 & 0.16 & 93 & 0.18 & - & - \\
\hline 5 & $\mathrm{XOS}-b-\mathrm{MeOl}$ & 1000 & 5 & 71 & 0.16 & 77 & 0.17 & 95 & 110 \\
\hline 6 & XOS- $b$-MeRic & 200 & 1 & 177 & 0.14 & 389 & 0.02 & 184 & 230 \\
\hline 7 & XOS- $b$-MeRic & 400 & 2 & 85 & 0.04 & 143 & 0.10 & - & - \\
\hline 8 & XOS- $b$-MeRic & 600 & 3 & 66 & 0.08 & 79 & 0.11 & - & - \\
\hline 9 & XOS- $b$-MeRic & 800 & 4 & 61 & 0.10 & 79 & 0.13 & - & - \\
\hline 10 & XOS- $b$-MeRic & 1000 & 5 & 62 & 0.23 & 75 & 0.06 & 65 & 68 \\
\hline
\end{tabular}

Table 3 Summary of the parameters employed for performing the microfluidics experiments and the sizes obtained by DLS at $90^{\circ}$ using Malvern and multi-angle DLS and SLS using the ALV for the sample XOS- $b$-MeOl dispersed in THF at a concentration of $2 \mathrm{mg} / \mathrm{mL}$ then filtered using a $0.45 \mu \mathrm{m}$ PTFE filter. 


\section{Supporting Information for:}

\section{Synthesis and Self-Assembly of Xylan-Based Amphiphiles:}

\section{from Bio-Based Vesicles to Antifungal Properties}

Julien Rosselgong ${ }^{1,2}$, Cédric Cabral Almada ${ }^{1,2}$, Gauvin Hemery ${ }^{1,2}$, Maud Chemin ${ }^{1,2}$,JeanMichel Guigner ${ }^{3}$, Guillaume Chollet ${ }^{4}$, Gilles Labat ${ }^{5}$, Denilson Da Silva Perez ${ }^{5}$, Frédérique Ham-Pichavant ${ }^{1,2}$, Etienne Grau ${ }^{1,2}$, Stéphane Grelier ${ }^{1,2^{*}}$, Sébastien Lecommandoux ${ }^{1,2^{*}}$, Henri Cramail $^{1,2^{*}}$

${ }^{1}$ CNRS, LCPO, UMR 5629, 16 av. Pey Berland, Pessac, F-33600, France

${ }^{2}$ Univ. Bordeaux, LCPO, UMR 5629, 16 av. Pey Berland, Pessac, F-33600, France

${ }^{3}$ Institut de Minéralogie, de Physique des Matériaux et de Cosmochimie (IMPMC), Sorbonne Universities - UPMC University Paris 06, UMR CNRS 7590, MNHN, IRD UR 206, 75252 Paris cedex 05, France

${ }^{4}$ ITERG, 11 rue Gaspard Monge, Parc Industriel, Pessac cedex, F-33600, France ${ }^{5}$ FCBA, New Materials Division, Domaine Universitaire, Grenoble cedex9, F-38044, France

Dilute acidic hydrolysis of xylan. A scale up experiment, times 120 for the comparison of mass of MGX involved, was performed compared to the acidic hydrolysis described previously [1-2]. $120 \mathrm{~g}$ of ultrafiltrated MGX were beforehand solubilized in $1.2 \mathrm{~L}$ of deionized water and sonicated for $30 \mathrm{~min}$. $1.2 \mathrm{~L}$ of a $1.4 \mathrm{M}$ solution of $\mathrm{H}_{2} \mathrm{SO}_{4}$ was placed in a $5 \mathrm{~L}$ reactor equipped with a condenser and a double jacket temperature regulating system set up to $90^{\circ} \mathrm{C}$ under mechanical stirring. When the acidic solution in the reactor reached $90^{\circ} \mathrm{C}$, the MGX solution was added and let react for $45 \mathrm{~min}$. The reaction mixture was then 
transferred in a $4 \mathrm{~L}$ beaker and cooled down with an ice bath. It was then split in three $4 \mathrm{~L}$ beakers and neutralized using 7.2 $\mathrm{L}$ of saturated $\mathrm{Ba}(\mathrm{OH})_{2}$ solution. Finally, centrifugation (3500 rpm, RT, $15 \mathrm{~min}$ ) was conducted to separate the precipitated salt from the supernatant, which was then concentrated by rotary evaporation, and lastly freeze dried. The xylooligosaccharides (XOS) obtained were purified by precipitation in EtOH: the hydrolysis product was solubilized in $0.17 \mathrm{~L}$ of deionized water then precipitated in $3 \mathrm{~L}$ of EtOH to produce a 95 vol.\% of EtOH: $\mathrm{H}_{2} \mathrm{O}$. This mixture was allowed to stir for $1 \mathrm{~h}$ prior centrifugation (3500 rpm, RT, $15 \mathrm{~min}$ ). The XOS precipitated were re-dissolved in water before freeze-drying. An amount of $75.25 \mathrm{~g}$ of pale brown powder was collected for an overall yield of XOS of $61.9 \%$, the potential structure of the XOS obtained is shown in Scheme S1.

Dilute acidic hydrolysis of xylan. Recently, Chemin et al. reported the production of welldefined XOS by mild sulfuric acid hydrolysis of beechwood xylans [2]. The authors studied different experimental parameters such as the hydrolysis temperature, its reaction time and the concentration of the dilute acidic media: these parameters were demonstrated to play a crucial role in the acidic hydrolysis and varying them enable to tune the final composition of the XOS, especially their molecular weight and the amount of 4-O-methylglucuronic acid (MeGlcA) substituent. An optimum set of conditions (i.e. $90^{\circ} \mathrm{C}, 45 \mathrm{~min}, 0.7 \mathrm{M} \mathrm{H}_{2} \mathrm{SO}_{4}$ ) was found to be repeatable allowing the production of XOS containing six xylose units with only one MeGlcA as an average, as shown in Scheme S1, for an expected molar mass of 1,000 g.mol ${ }^{-1}$. In the present study, we have followed this set of conditions while scaling up the acidic hydrolysis from $500 \mathrm{mg}$ of ultrafiltrated xylans (the initial raw material needs to be purified by ultrafiltration to remove a population of aggregated xylans using tangential flow filtration with a $0.45 \mu \mathrm{m}$ pore size membrane) to 120 grams (in testing 5 grams and 20 grams scale in between) of this starting material in order to obtain a large batch of hydrophilic segment which would be the foundation of the elaboration of amphiphilic bioconjugates. The XOS produced were characterized by size exclusion chromatography (SEC), ${ }^{\mathrm{I}} \mathrm{H},{ }^{13} \mathrm{C}$ and HSQC NMR spectroscopies and matrix-assisted laser desorption/ionization time-of-flight (MALDI-TOF) analyses, as shown in Figure S1-S5. The SEC analysis presented in Figure S1 demonstrates the decrease of molecular weight from the MGX to the XOS after acidic hydrolysis. The retention time on the chromatogram shifts from $29.1 \mathrm{~min}$ for the MGX to 34.9 min for the XOS and the molecular weight obtained, based on a pullulan calibration, decreases from $M_{n}=10,700$ g.mol ${ }^{-1}$ with $Ð=1.75$ for the MGX to $M_{n}=670$ g.mol ${ }^{-1}$ with $Ð=$ 
2.73 for the XOS. From the NMR analyses (Figure S2-S4), it could be deduced that the XOS were composed of a linear backbone of $\beta-(1-4)$-D-xylose mono substituted in C-2 with a MeGlcA unit [1]. However the MALDI-TOF spectrum presented in Figure S5 gives a better insight on the structure of the XOS: two main populations can be observed, the minor one with no MeGlcA branching unit with a $\mathrm{DP}_{\mathrm{n}}$ of xylose units varying from 3 to 9 and the major one with one MeGlcA branching unit and with a $\mathrm{DP}_{\mathrm{n}}$ of xylose units varying from 3 to 12 .

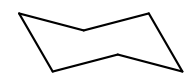

Supporting Scheme S1. General structure of the xylo-oligosaccharides obtained after acidic hydrolysis.

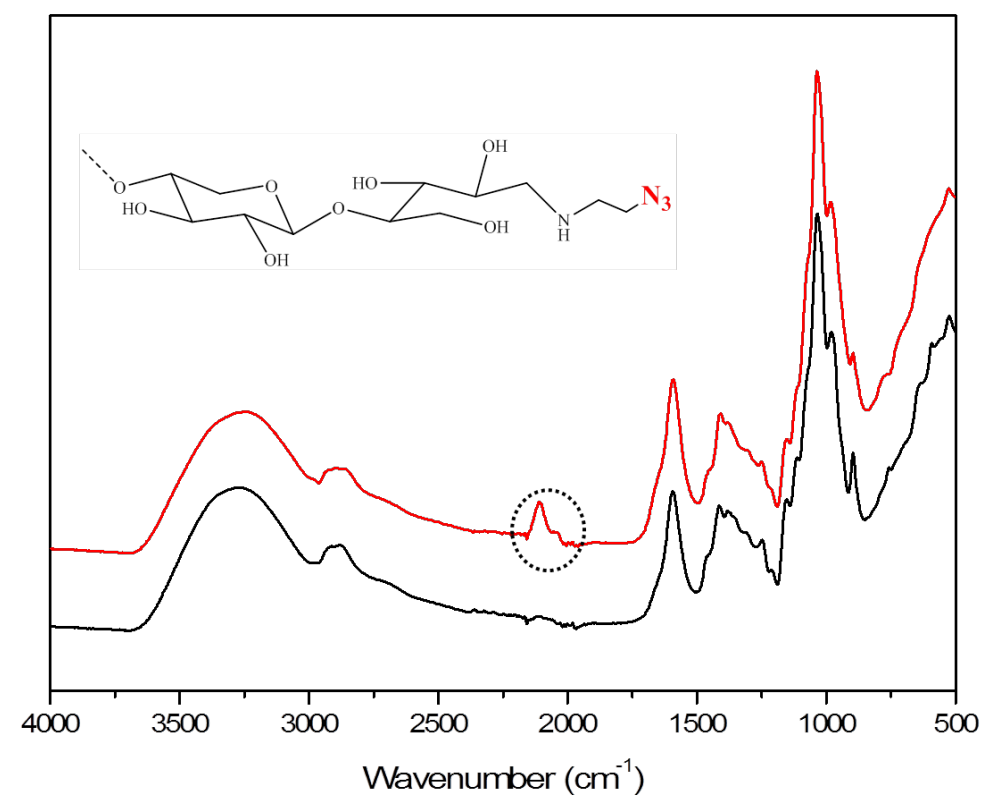

Supporting Figure S1 Infra red spectra of xylo-oligosaccharides before (black) and after (red) reductive amination with 2-aminoethyl chloride followed by reaction with sodium azide. 


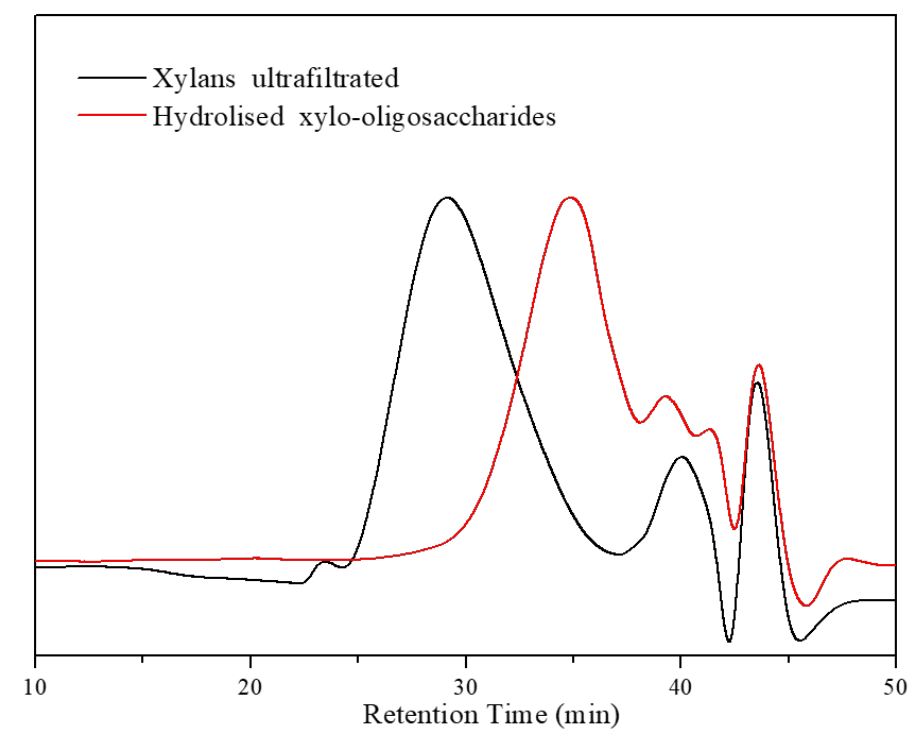

Supporting Figure S2. SEC traces recorded for a native xylan after ultrafiltration and xylooligosaccharides after hydrolysis acid using a refractive index detector calibrated with pullulans standards.

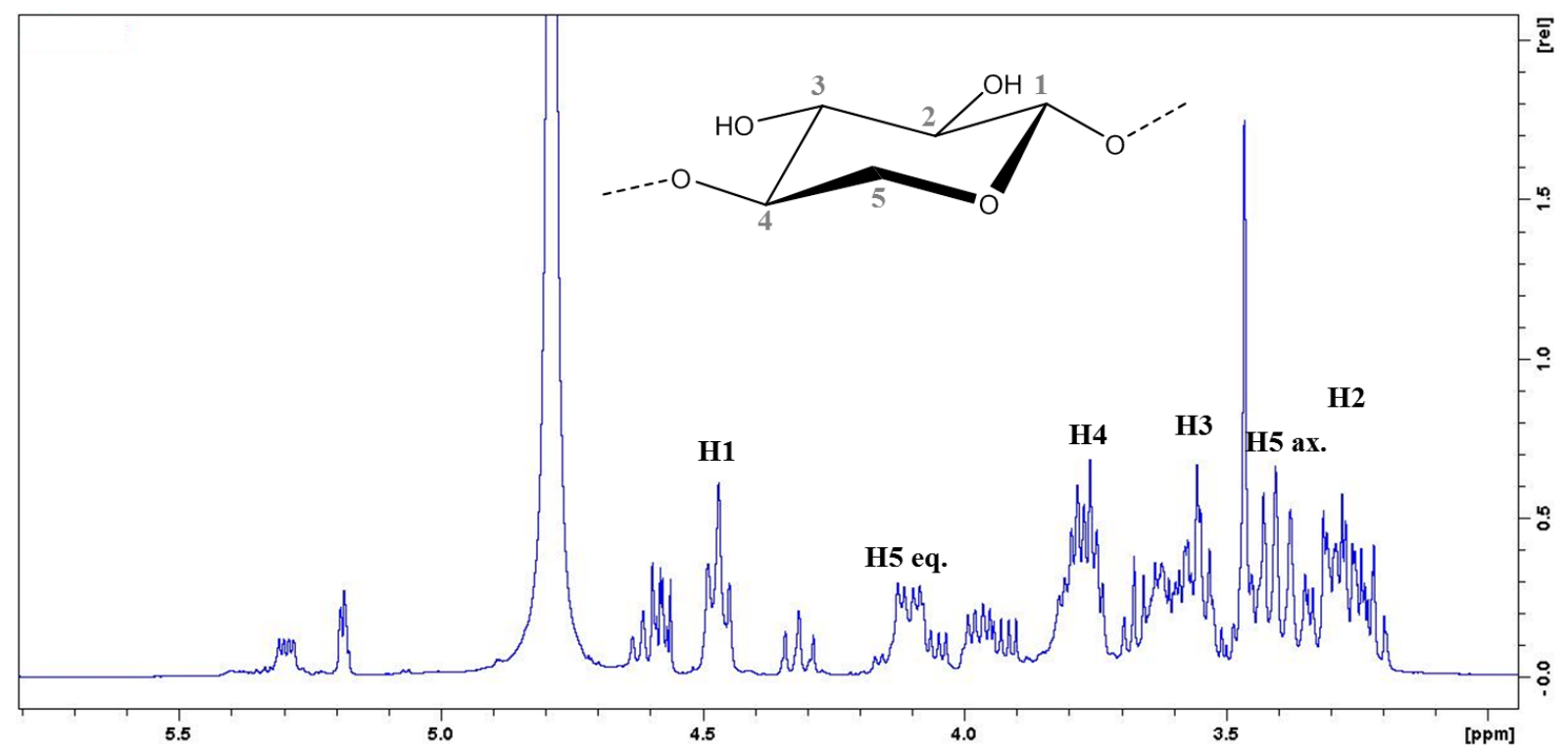

Supporting Figure S3. ${ }^{1} \mathrm{H}$ NMR spectrum in $\mathrm{D}_{2} \mathrm{O}$ of the xylo-oligosaccharide obtained after acidic hydrolysis. 


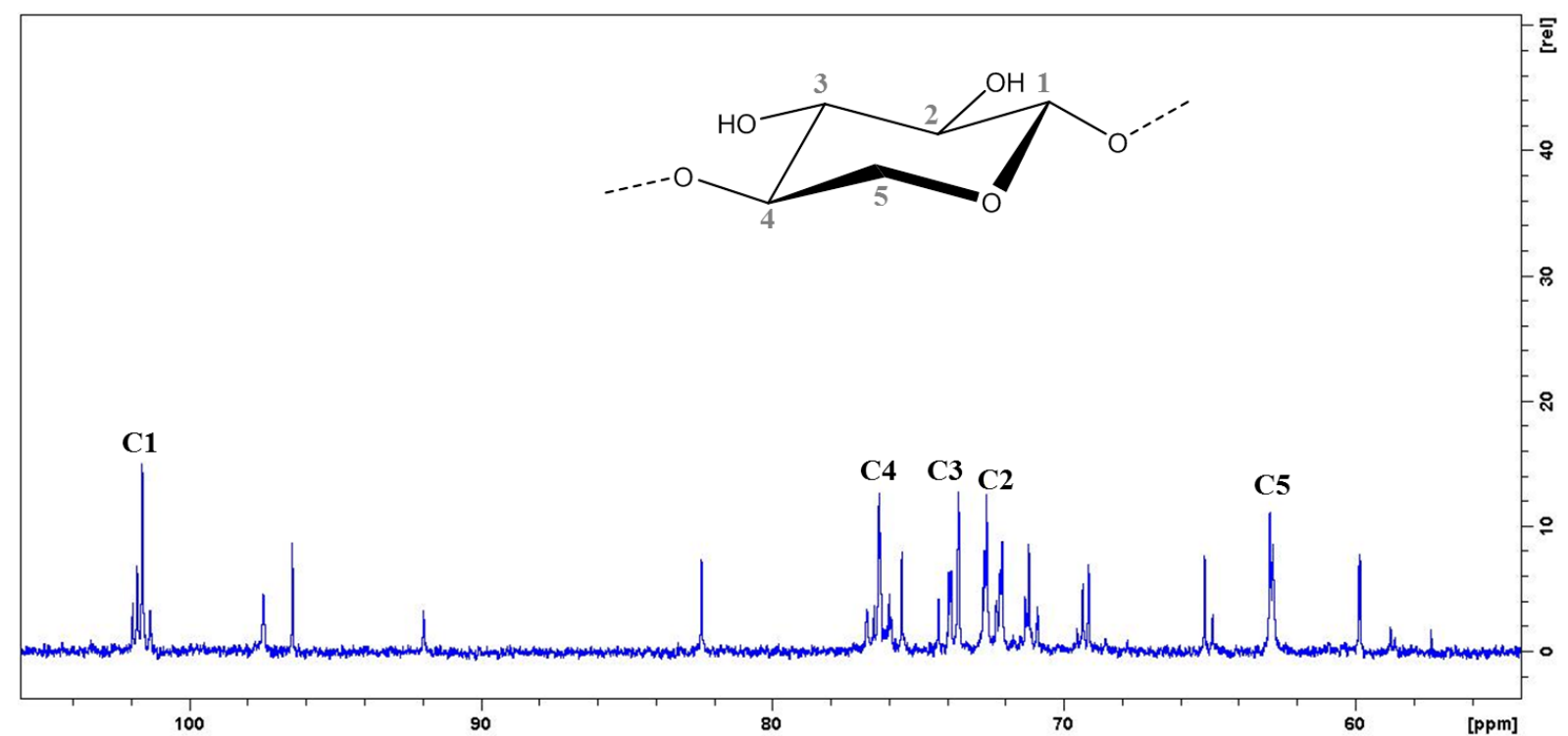

Supporting Figure S4. ${ }^{13} \mathrm{C}$ NMR spectrum in $\mathrm{D}_{2} \mathrm{O}$ of the xylo-oligosaccharide obtained after acidic hydrolysis.

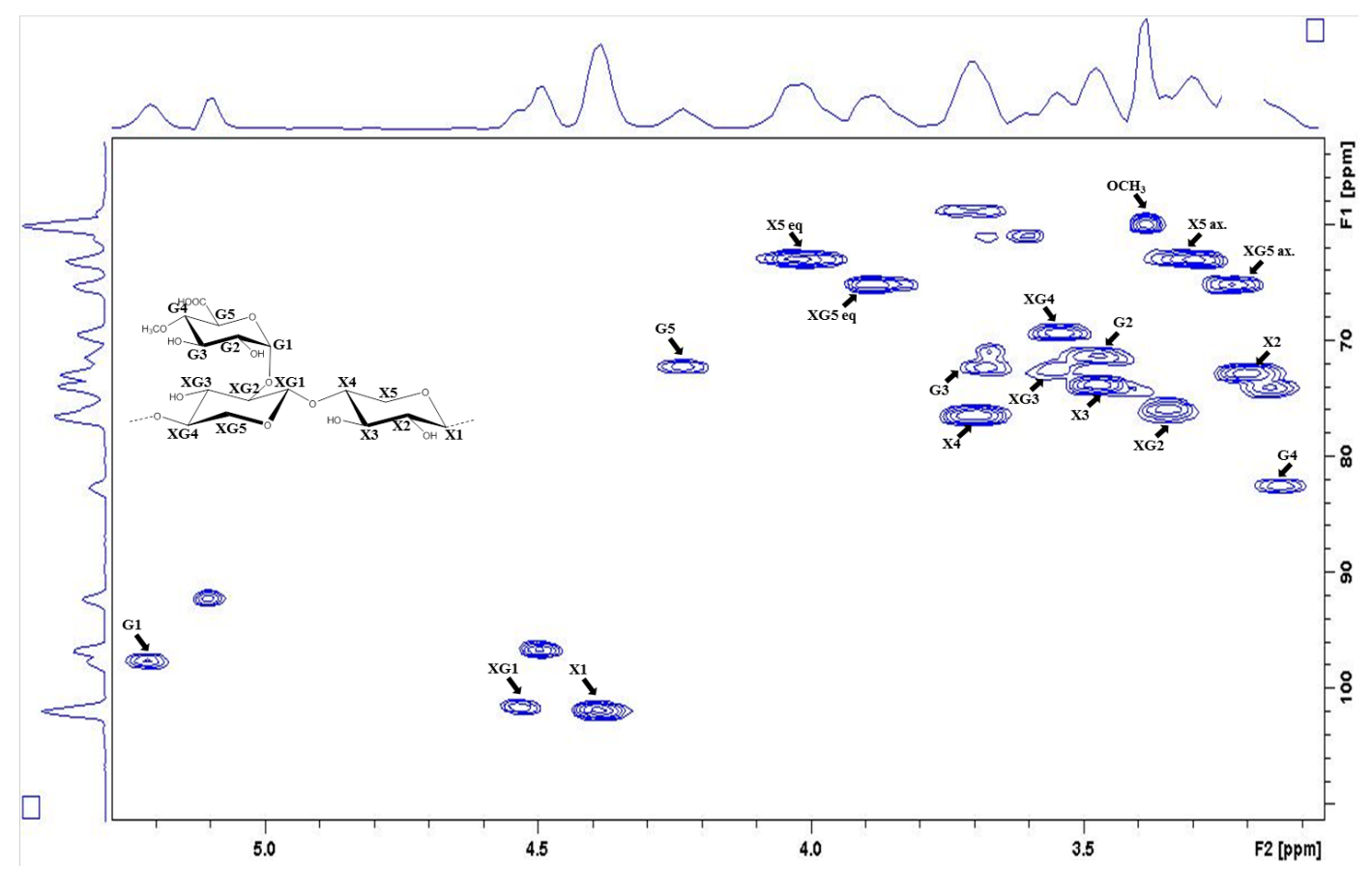

Supporting Figure S5. HSQC NMR spectrum in $\mathrm{D}_{2} \mathrm{O}$ of the xylo-oligosaccharide obtained after acidic hydrolysis. 


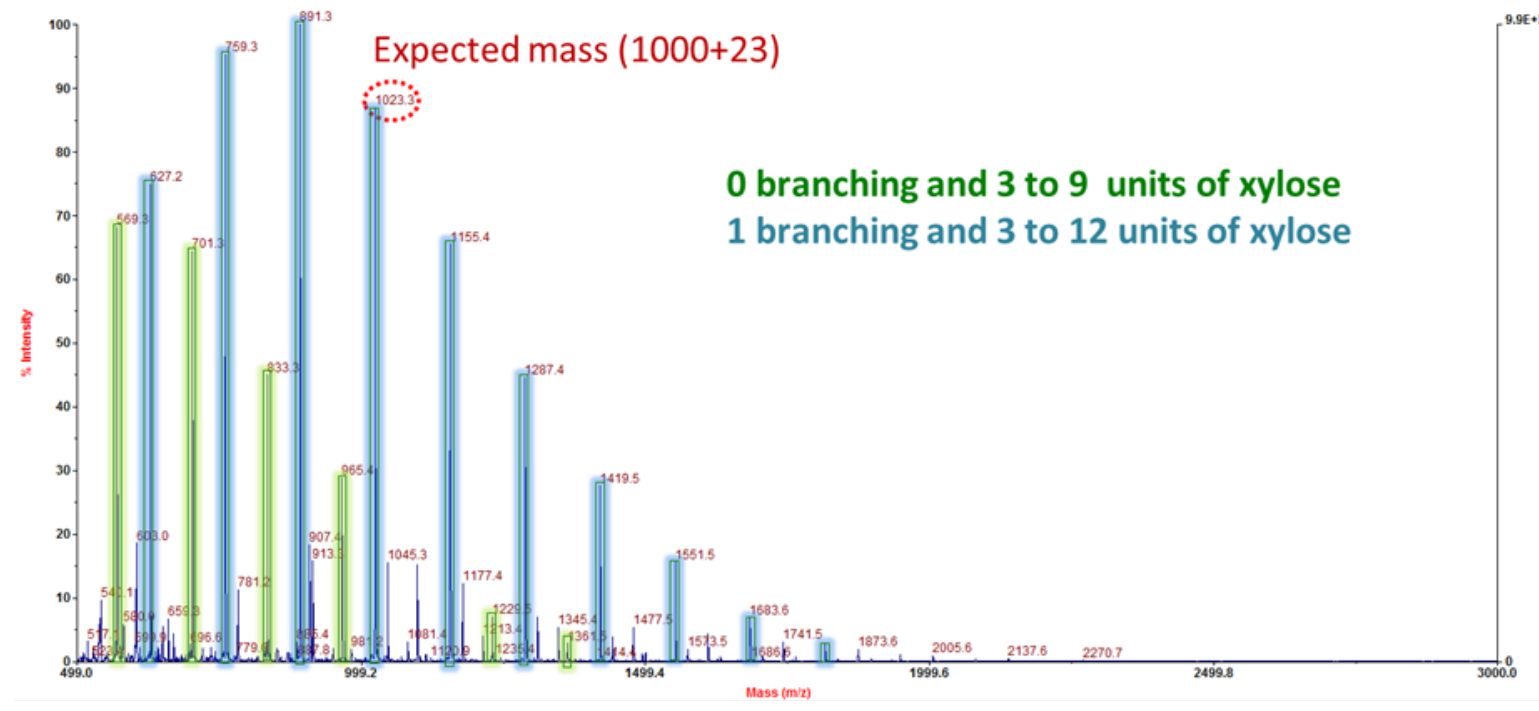

Supporting Figure S6. MALDI-TOF spectrum of the xylo-oligosaccharides obtained after acidic hydrolysis.

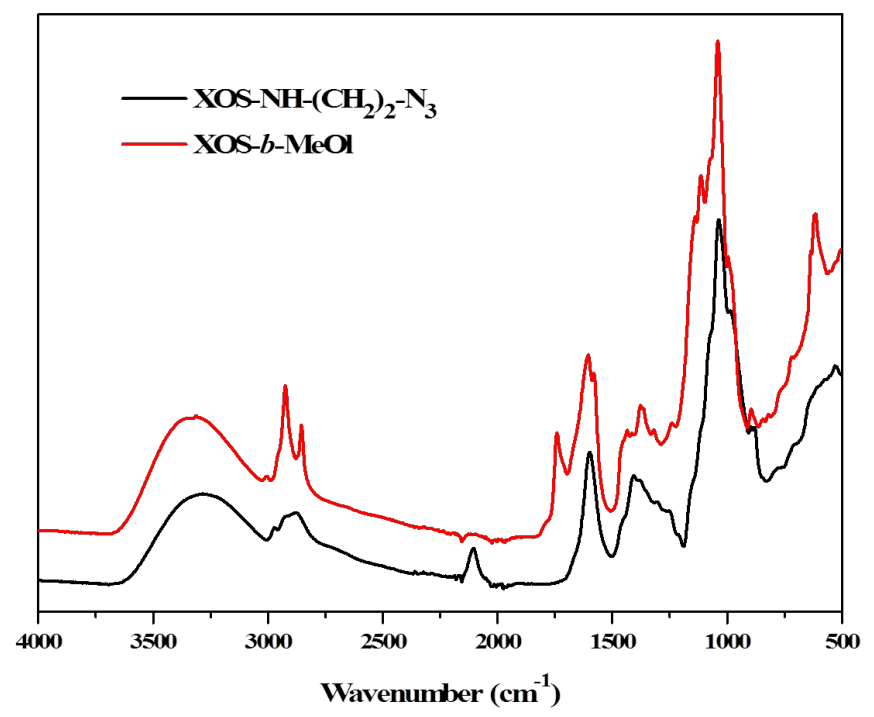

Supporting Figure S7. Overlaid FTIR spectra of the XOS-NH- $\left(\mathrm{CH}_{2}\right)_{2}-\mathrm{N}_{3}$ and XOS- $b-\mathrm{MeOl}$ after 'click' reaction. 


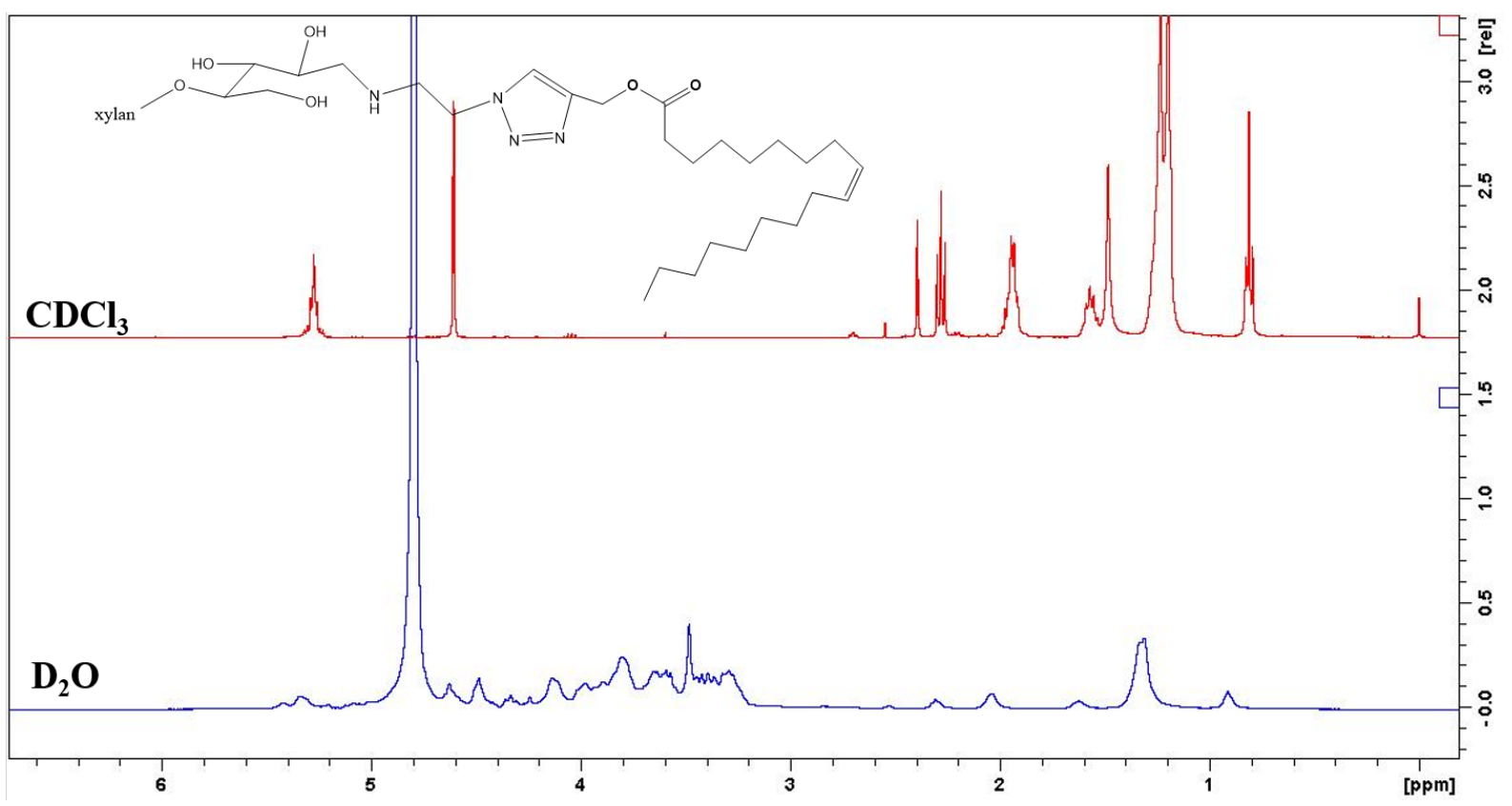

Supporting Figure S8. Overlaid spectra of the ${ }^{1} \mathrm{H}$ NMR of the bioconjugate XOS- $b-\mathrm{MeO}$ in $\mathrm{CDCl}_{3}$ (top) and in $\mathrm{D}_{2} 0$ (bottom).

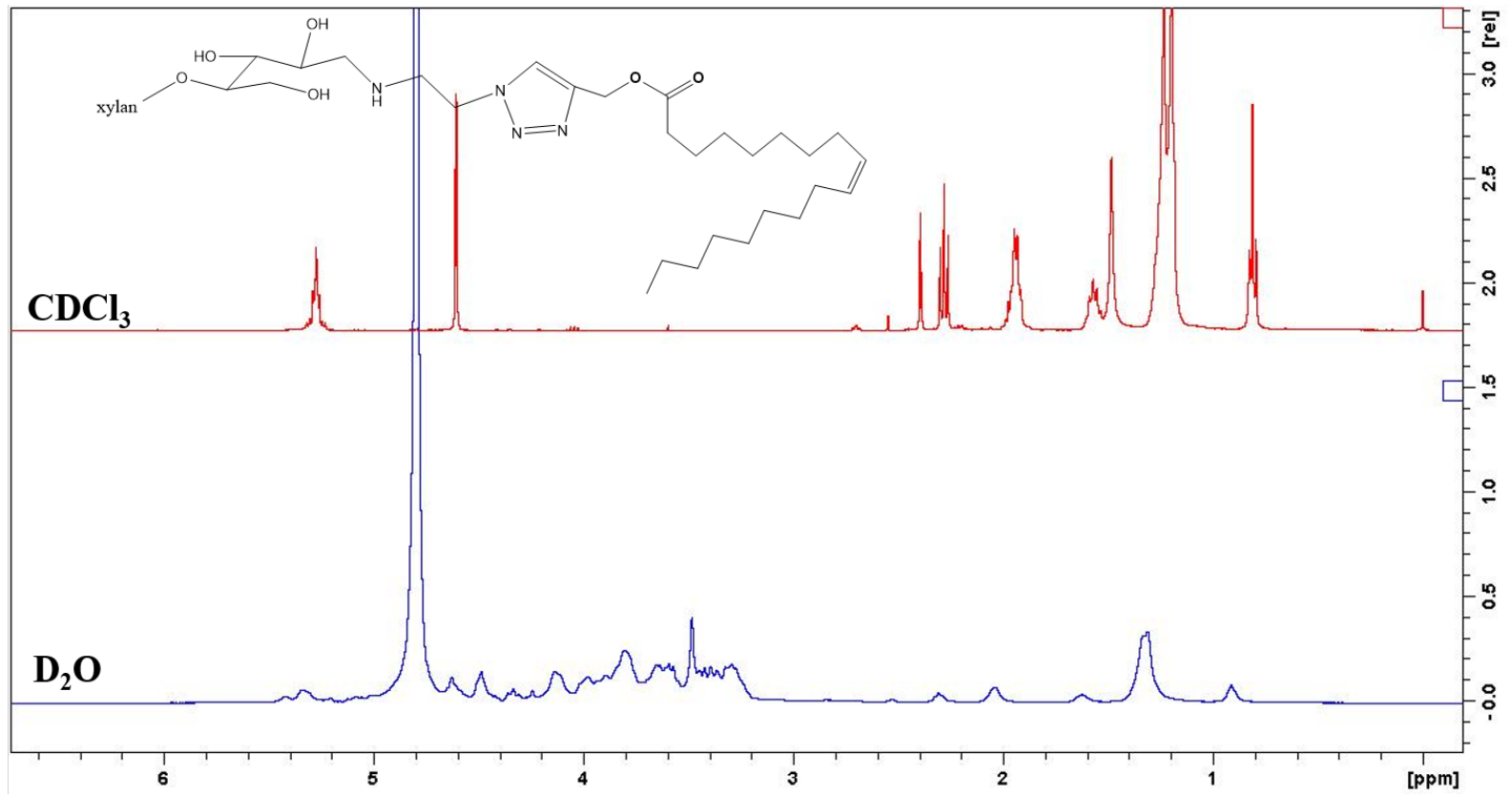

Supporting Figure S9. Overlaid spectra of the ${ }^{1} \mathrm{H}$ NMR of the bioconjugate XOS- $b$-MeRic in $\mathrm{CDCl}_{3}$ (top) and in $\mathrm{D}_{2} \mathrm{O}$ (bottom). 


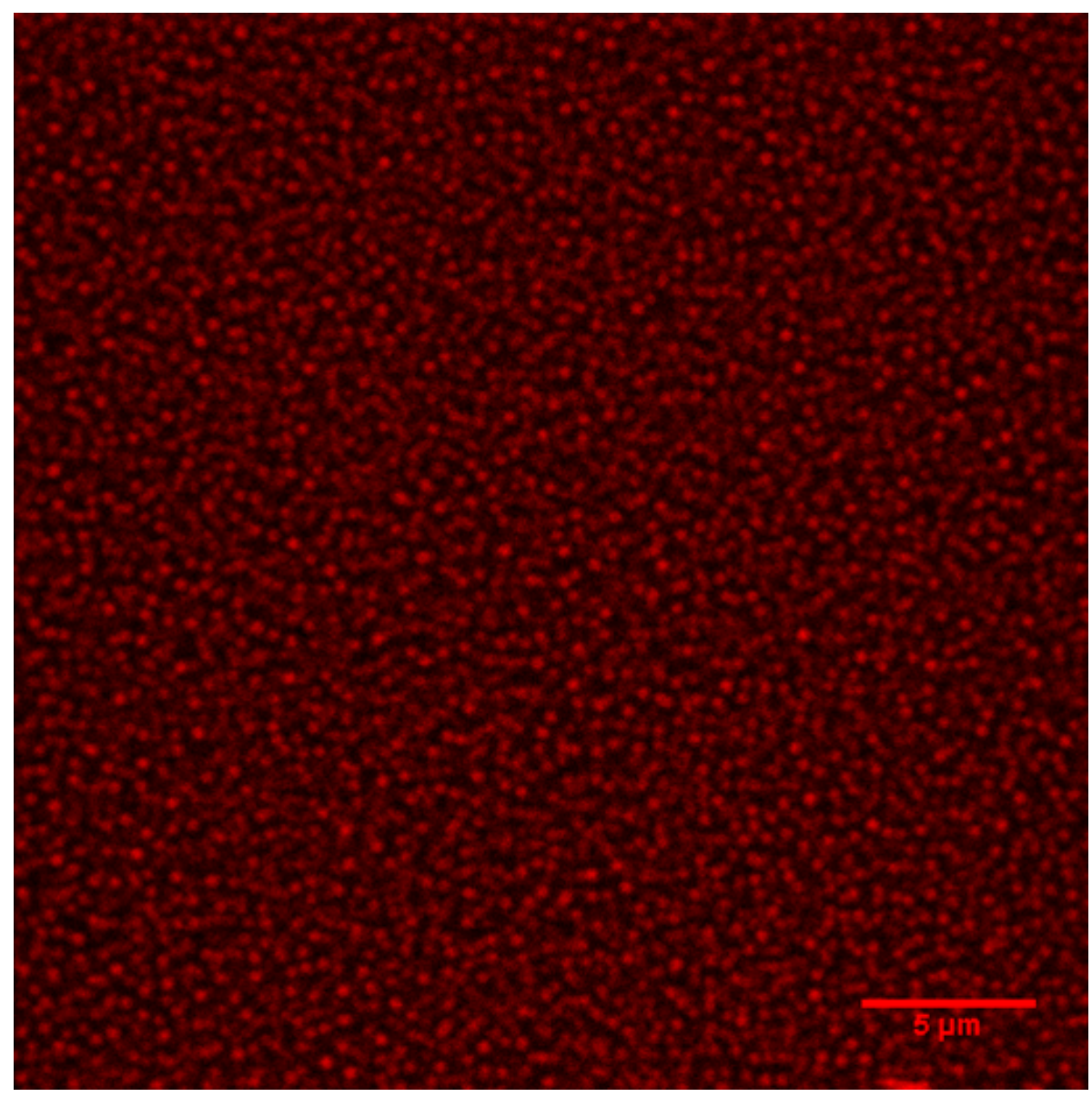

Supporting Figure S10. Confocal microscope image of nile red loaded vesicles obtained from the self-assembly of the bioconjugate XOS- $b$-MeOl by microfluidics.

\begin{tabular}{|c|c|c|c|}
\hline \multicolumn{2}{|c|}{ Direct solubilization in $\mathbf{H}_{2} \mathbf{O}$} & \multicolumn{2}{c|}{ DLS at 90 } \\
\hline Sample & $\begin{array}{c}\text { Concentration } \\
(\mathbf{m g} / \mathbf{m L})\end{array}$ & $\begin{array}{c}\mathbf{R}_{\mathbf{H}} \\
(\mathbf{n m})\end{array}$ & PDI \\
\hline XOS- $b$-MeO1 & 10 & 105 & 0.176 \\
\hline XOS- $b$-MeO1 & 0.1 & 106 & 0.193 \\
\hline XOS- $b$-MeRic & 10 & 88 & 0.221 \\
\hline XOS- $b$-MeRic & 0.1 & 90 & 0.230 \\
\hline
\end{tabular}

Supporting Table S1. Summary of the sizes obtained by DLS at $90^{\circ}$ using Malvern for the samples XOS-b-MeOl and XOS- $b$-MeRic at different concentrations by direct solubilization in water. 


\begin{tabular}{|c|c|c|c|c|c|c|}
\hline \multicolumn{2}{|c|}{ Microfluidic } & \multicolumn{5}{|c|}{ In $\mathrm{THF} / \mathrm{H}_{2} \mathrm{O}$ mixture } \\
\hline Exp & Sample & $\mathbf{D}_{\mathrm{THF}}$ & $\mathbf{D}_{\mathrm{H} 20}$ & SR & $\begin{array}{r}\mathbf{R}_{\mathbf{H}} \\
(\mathrm{nm})\end{array}$ & PDI \\
\hline 11 & $\mathrm{XOS}-b-\mathrm{MeO}$ & 50 & 100 & 2 & 140 & 0.08 \\
\hline 12 & $\mathrm{XOS}-b-\mathrm{MeO}$ & 100 & 200 & 2 & 61 & 0.07 \\
\hline 13 & $\mathrm{XOS}-b-\mathrm{MeO}$ & 150 & 300 & 2 & 55 & 0.05 \\
\hline 14 & $\mathrm{XOS}-b-\mathrm{MeOl}$ & 200 & 400 & 2 & 88 & 0.07 \\
\hline 15 & $\mathrm{XOS}-b-\mathrm{MeOl}$ & 250 & 500 & 2 & 88 & 0.07 \\
\hline 16 & $\mathrm{XOS}-b-\mathrm{MeOl}$ & 300 & 600 & 2 & 68 & 0.07 \\
\hline 17 & $\mathrm{XOS}-b-\mathrm{MeOl}$ & 200 & 200 & 1 & 145 & 0.11 \\
\hline 18 & $\mathrm{XOS}-b-\mathrm{MeOl}$ & 200 & 400 & 2 & 88 & 0.08 \\
\hline 19 & $\mathrm{XOS}-b-\mathrm{MeOl}$ & 200 & 600 & 3 & 82 & 0.15 \\
\hline 20 & $\mathrm{XOS}-b-\mathrm{MeOl}$ & 200 & 800 & 4 & 75 & 0.16 \\
\hline 21 & XOS- $b-\mathrm{MeOl}$ & 200 & 1000 & 5 & 73 & 0.18 \\
\hline 22 & XOS- $b-\mathrm{MeOl}$ & 200 & 200 & 1 & 175 & 0.14 \\
\hline 23 & XOS- $b-\mathrm{MeOl}$ & 200 & 400 & 2 & 115 & 0.07 \\
\hline 24 & XOS- $b-\mathrm{MeOl}$ & 200 & 600 & 3 & 110 & 0.07 \\
\hline 25 & XOS- $b-\mathrm{MeO}$ & 200 & 800 & 4 & 85 & 0.16 \\
\hline 26 & XOS- $b-\mathrm{MeOl}$ & 200 & 1000 & 5 & 71 & 0.16 \\
\hline 27 & XOS- $b$-MeRic & 200 & 200 & 1 & 138 & 0.07 \\
\hline 28 & XOS- $b$-MeRic & 200 & 400 & 2 & 70 & 0.07 \\
\hline 29 & XOS- $b$-MeRic & 200 & 600 & 3 & 65 & 0.19 \\
\hline 30 & XOS- $b$-MeRic & 200 & 800 & 4 & 61 & 0.22 \\
\hline 31 & XOS- $b$-MeRic & 200 & 1000 & 5 & 58 & 0.18 \\
\hline 32 & XOS- $b$-MeRic & 200 & 200 & 1 & 150 & 0.05 \\
\hline 33 & XOS- $b$-MeRic & 200 & 400 & 2 & 74 & 0.07 \\
\hline 34 & XOS- $b$-MeRic & 200 & 600 & 3 & 70 & 0.14 \\
\hline 35 & XOS- $b$-MeRic & 200 & 800 & 4 & 64 & 0.14 \\
\hline 36 & XOS- $b$-MeRic & 200 & 1000 & 5 & 60 & 0.18 \\
\hline
\end{tabular}

Supporting Table S2 Summary of the parameters employed for performing the microfluidics experiments and the sizes obtained by DLS at $90^{\circ}$ using Malvern for the sample XOS- $b$-MeOl dispersed in THF at a concentration of $2 \mathrm{mg} / \mathrm{mL}$ then filtered using a $0.45 \mu \mathrm{m}$ PTFE filter. 


\section{Notes and references}

[1] M. Chemin, A. Rakotovelo, F. Ham-Pichavant, G. Chollet, D. da Silva Perez, M. PetitConil, H. Cramail, S. Grelier. Holzforschung. 2015, 69, 713-720.

[2] M. Chemin, A-L.Wirotius, F. Ham-Pichavant, G. Chollet, D. Da Silva Perez, M. PetitConil, H. Cramail, S. Grelier. European Polymer Journal. 2015, 66, 190-197. 